Draft VERsion August 17, 2021

Preprint typeset using $\mathrm{L}^{\mathrm{A}} \mathrm{T} \mathrm{E}$. style emulateapj v. 01/23/15

\title{
ENRICHMENT OF $R$-PROCESS ELEMENTS IN DWARF SPHEROIDAL GALAXIES IN CHEMO-DYNAMICAL EVOLUTION MODEL
}

\author{
Yutaka Hirai ${ }^{1,2,7}$, Yuhri Ishimaru ${ }^{3,4}$, Takayuki R. Saitoh ${ }^{5}$, Michiko S. Fujii $^{2}$, Jun Hidaka ${ }^{6,2}$, And \\ TOSHITAKa KAJINO 2,1 \\ ${ }^{1}$ Department of Astronomy, Graduate School of Science, the University of Tokyo, 7-3-1 Hongo, Bunkyo-ku, Tokyo 113-0033, Japan \\ ${ }^{2}$ Division of Theoretical Astronomy, National Astronomical Observatory of Japan, 2-21-1 Osawa Mitaka, Tokyo 181-8588, Japan; \\ yutaka.hirai@nao.ac.jp \\ ${ }^{3}$ Department of Material Science, International Christian University, 3-10-2 Osawa, Mitaka, Tokyo 181-8585, Japan \\ ${ }^{4}$ Institut d'Astrophysique de Paris, 98bis Boulevard Arago, 75014, Paris, France \\ ${ }^{5}$ Earth-Life Science Institute, Tokyo Institute of Technology, 2-12-1 Ookayama, Meguro-ku, Tokyo 152-8551, Japan \\ ${ }^{6}$ School of Science and Engineering, Meisei University, 2-1-1 Hodokubo, Hino, Tokyo 191-0042, Japan \\ Draft version August 17, 2021
}

\section{ABSTRACT}

The rapid neutron-capture process ( $r$-process) is a major process to synthesize elements heavier than iron, but the astrophysical site(s) of $r$-process is not identified yet. Neutron star mergers (NSMs) are suggested to be a major $r$-process site from nucleosynthesis studies. Previous chemical evolution studies however require unlikely short merger time of NSMs to reproduce the observed large starto-star scatters in the abundance ratios of $r$-process elements relative to iron, [Eu/Fe], of extremely metal-poor stars in the Milky Way (MW) halo. This problem can be solved by considering chemical evolution in dwarf spheroidal galaxies (dSphs) which would be building blocks of the MW and have lower star formation efficiencies than the MW halo. We demonstrate that enrichment of $r$-process elements in dSphs by NSMs using an $N$-body/smoothed particle hydrodynamics code. Our highresolution model reproduces the observed [Eu/Fe] by NSMs with a merger time of 100 Myr when the effect of metal mixing is taken into account. This is because metallicity is not correlated with time up to $\sim 300$ Myr from the start of the simulation due to low star formation efficiency in dSphs. We also confirm that this model is consistent with observed properties of dSphs such as radial profiles and metallicity distribution. The merger time and the Galactic rate of NSMs are suggested to be $\lesssim 300$ Myr and $\sim 10^{-4} \mathrm{yr}^{-1}$, which are consistent with the values suggested by population synthesis and nucleosynthesis studies. This study supports that NSMs are the major astrophysical site of $r$-process. Subject headings: galaxies: abundances — galaxies: dwarf — galaxies: evolution — Local Group — methods: numerical

\section{INTRODUCTION}

Elements heavier than iron are mainly synthesized by the rapid neutron capture process ( $r$-process) as well as the slow neutron capture and proton capture processes. More than $90 \%$ of the number of elements such as europium $(\mathrm{Eu})$, gold $(\mathrm{Au})$, and platinum $(\mathrm{Pt}) \mathrm{T}$ in the solar system are synthesized by $r$-process (Burris et al. 2000). Sufficiently neutron-rich environment is required in order to synthesize $r$-process elements with mass number $(A)$ over 110.

The observed $r$-process elemental abundance ratios, such as $[\mathrm{Eu} / \mathrm{Fe}]$, in extremely metal-poor (EMP) stars $([\mathrm{Fe} / \mathrm{H}] \lesssim-3)$ show large star-to-star scatters. These scatters are seen in EMP stars in the Milky Way (MW) halo as well as the Local Group (LG) dwarf spheroidal galaxies (dSphs) (e.g., Woolf et al. 1995; McWilliam et al. 1995; Rvan et al. 1996; Shetrone 1996; McWillian 1998; Westin et al. 2000; Burris et al. 2000; Fulbright 2000; Norris et al. 2001; Johnson 2002; Francois et al. 2003; Honda et al. 2004; Francois et al. 2007; Sneden et al. 2008; Tolstov et al. 2009; Frebel et al. 2010a,b; Letarte et al. 2010;

\footnotetext{
${ }^{7}$ Research Fellow of Japan Society for the Promotion of Science

$\dagger$ Hereafter, we call these elements $r$-process elements

$\ddagger[\mathrm{A} / \mathrm{B}]=\log _{10}\left(N_{\mathrm{A}} / N_{\mathrm{B}}\right)-\log _{10}\left(N_{\mathrm{A}} / N_{\mathrm{B}}\right) \odot$, where $N_{\mathrm{A}}$ and $N_{\mathrm{B}}$ are number densities of elements $\mathrm{A}$ and $\mathrm{B}$, respectively.
}

Aoki et al. 2013; Ishigaki et al. 2013). The mechanism of star-to-star scatters in the abundance studies should be clarified simultaneously with the astrophysical site(s) of $r$-process.

Neutrino-driven winds from proto-neutron stars of core-collapse supernovae (CCSNe) have long been regarded as one of the possible sites of $r$-process (e.g., Mever et al. 1992; Wooslev et al. 1994; Qian \& Wooslev 1996; Wanaio et al. 2001). Previous chemical evolution studies suggest that the observed $[\mathrm{Eu} / \mathrm{Fe}]$ scatter is well reproduced by models assuming that CCSNe of low-mass $\left(8-10 M_{\odot}\right)$ progenitors produce $r$-process elements (e.g., Mathews et al. 1992; Ishimaru \& Wanaio 1999; Travaglio et al. 1999; Tsujimoto et al. 2000; Travaglio et al. 2001; Ishimaru et al. 2004; Argast et al. 2004). However, recent hydrodynamical simulations of CCSNe, which include neutrino transport in sophisticated manner, suggest that the PNS winds of CCSNe do not necessarily produce a neutron-rich condition suitable for $r$-process (e.g., Reddy et al. 1998; Roberts 2012; Martínez-Pinedo et al. 2012; Roberts et al. 2012; Horowitz et al. 2012). Nucleosynthesis calculations suggest that heavy elements with $A \gtrsim 110$, are difficult to be synthesized in CCSNe due to such too weak neutron-rich environments (e.g., Wanajo et al. 2011; Wanajo 2013).

Binary neutron star mergers (NSMs) are also suggested 
to be a promising site of $r$-process (Lattimer \& Schramm 1974, 1976; Lattimer et al. 1977; Svmbalisty \& Schramm 1982; Eichler et al. 1989; Mever 1989). Recent detailed nucleosynthesis calculations show that heavy $r$-process elements are successfully synthesized in NSMs (Freiburghaus et al. 1999; Goriely et al. 2011; Korobkin et al. 2012; Bauswein et al. 2013; Rosswog et al. 2014; Wanajo et al. 2014). In addition, near infrared afterglow of the Swift short gamma-ray burst, GRB130603B (Berger et al. 2013; Tanvir et al. 2013) is detected. This is suggested to be a piece of evidence that progenitors of short gamma-ray bursts are compact binary mergers and $r$-process nucleosynthesis occurs there (Tanaka \& Hotokezaka 2013).

NSMs have the long merger time and the low rate. The neutron star (NS) binaries lose their energy very slowly due to the gravitational emissions. The merger time is estimated $\gtrsim 100 \mathrm{Myr}$ from observed binary pulsars (Lorimer 2008). Recent predictions from population synthesis models also suggest that most of NS binaries merge $\gtrsim 100$ Myr after their formation (Dominik et al. 2012). On the other hand, the NSM rate is estimated to be $10^{-6}-10^{-3} \mathrm{yr}^{-1}$ for a MW size galaxy from the observed binary pulsars (Abadie et al. 2010a).

However, early studies of galactic chemical evolution pointed out that it is difficult to reproduce the observed trend of $[\mathrm{Eu} / \mathrm{Fe}]$ of EMP stars by NSMs due to their long merger time and the low rate Mathews \& Cowan 1990; Argast et al. 2004). Most of other recent studies also conclude that short merger time $(\lesssim 10 \mathrm{Myr})$ or a second site of $r$-process such as jet-like explosions of magnetorotational CCSNe (e.g., Winteler et al. 2012; Nishimura et al. 2015) is required to account for large star-to-star scatters in EMP stars (Matteucci et al. 2014; Komiva et al. 2014; Tsujimoto \& Shigeyama 2014; Cescutti et al. 2015; Wehmever et al. 2015). On the other hand, detailed population synthesis calculations suggest that production of NSMs with short-merger time ( $\sim 10 \mathrm{Myr})$ highly depends on the treatment of common envelop phase which is not well understood (e.g. Portegies Zwart \& Yungelson 1998; Dominik et al. 2012; Kinugawa et al. 2014). Dominik et al. (2012) suggest that NSMs with merger time of $\lesssim 10$ Myr cannot be produced in their most pessimistic model assuming each common envelop with an Hertzsprung gap donor causes a merger (Submodel B in Dominik et al. 2012). In addition, there are no observational clues that exist binary pulsars which merge within $\sim 10 \mathrm{Myr}$ so far (Lorimer 2008).

This discrepancy may be solved, if the Galactic halo is formed via mergers of sub-halos within the framework of hierarchical structure formation scenario (Ishimaru et al. 2015). Ishimaru et al. (2015) calculate the enrichment of $r$-process elements by NSMs with merger time of $100 \mathrm{Myr}$ (95\% of NSMs) and $1 \mathrm{Myr}(5 \%$ of NSMs) in their one-zone chemical evolution model for each sub-halo. They suggest that $[\mathrm{Eu} / \mathrm{Fe}]$ increases at $[\mathrm{Fe} / \mathrm{H}] \leq-3$ if the star formation efficiencies are lower in less massive sub-halos. According to their calculations, the observed scatters in $[\mathrm{Eu} / \mathrm{Fe}]$ in metal-poor stars are possibly explained by NSMs with merger time of 100 Myr. Key factors of chemical evolution such as the time variation of the star formation rate (SFR), outflow, and inflow strongly depend on thermodynamical feedback from SNe. Detailed chemo-dynamical evolution studies in low-mass galaxies like sub-halos are highly desirable to justify their assumptions to describe the enrichment history of $r$-process elements in a self-consistent manner between dynamical and chemical evolution of galaxies.

Recent hydrodynamical studies have performed a series of simulations of galaxy formation assuming that the NSMs are a major site of $r$-process (van de Voort et al. 2015; Shen et al. 2015). van de Voort et al. (2015) suggest that gas mixing processes such as galactic winds and hydrodynamic flows play the important roles to explain the observed Galactic $r$-process ratio. Their high-resolution model with $7.1 \times 10^{3} M_{\odot}$ of the mass of one gas particle $\left(m_{\text {gas }}\right)$ is, however, difficult to reproduce the observed $r$-process abundance ratios. They imply that additional metal mixing is required to explain the observation. Shen et al. (2015) also suggest that the observed $r$-process abundance ratios are possibly taken into account in their NSM models if the metal mixing in star-forming region is implemented. However, the mass resolution $\left(m_{\text {gas }}=2.0 \times 10^{4} M_{\odot}\right)$ in their models is as low as the fiducial low-resolution model of van de Voort et al. $(2015)\left(m_{\text {gas }}=5.7 \times 10^{4} M_{\odot}\right)$. It is therefore important to demonstrate that if the NSM models account for the observations in independent simulations of much higher resolution.

In this paper, we calculate enrichment of $r$-process elements ejected by NSMs in low mass galaxies with high-resolution $\left(m_{\text {gas }}=4.0 \times 10^{2} M_{\odot}\right)$ chemo-dynamical evolution models. We perform $N$-body/smoothed particle hydrodynamics (SPH) simulations of dSph models using ASURA code (Saitoh et al. 2008, 2009). We discuss effects of metal mixing in star-forming region as well as the dependence on the SFR, the merger time, and the rate of NSMs.

In $\S 2$, we introduce our code and models. In $\S 3$, we compare model predictions and observed properties of dSphs generated by our models. In $\S 4$, we discuss enrichment of $r$-process elements in dSphs. Finally in $\S 5$, we summarize our main results.

\section{METHOD AND MODELS}

\subsection{N-body / smoothed particle hydrodynamics code,} ASURA

We perform a series of simulations using an $N$-body / SPH code, ASURA (Saitoh et al. 2008, 2009). We adopt three different kinds of particles in our simulations: dark matter, gas, and star particles. We treat dark matter and star particles as collisionless particles. Dark matter particles contribute to the dynamical evolution of our model galaxies. Star particles mainly contribute to feedback of energy and heavy elements produced by CCSNe. We solve the hydrodynamical evolution of gas particles using SPH (e.g., Lucy et al. 1977; Gingold \& Monaghan 1977; Monaghan \& Lattanzio 1985; Monaghan et al. 1992).

Here we describe implementation of gravity and hydrodynamics in ASURA. Gravity is calculated by a treecode (Barnes \& Hut 1986) with GRAPE method (Makino et al.|1991) using the Phantom-GRAPE library (Tanikawa et al. 2012). Hydrodynamics is computed using a standard SPH method. For integration of selfgravitating fluid system, we adopt the fully asynchronous 
split time-integrator (FAST) algorithm in order to reduce calculation cost (Saitoh \& Makino 2010). We use the time-step limiter, which forces the timestep difference among neighbor particles to be less than four times long, in order to follow the evolution of strong shock regions such as supernova (SN) remnants (Saitoh \& Makino 2009). We use a metallicity dependent cooling/heating function generated by Cloudy (Ferland et al. 1998, 2013). The cooling/heating function covers the temperature range from $10 \mathrm{~K}$ to $10^{9} \mathrm{~K}$.

We allow star formation when gas particles satisfy three conditions. (1) Gas particles are conversing $(\nabla \cdot \mathbf{v}<$ $0)$. (2) The density is higher than threshold density, $n_{\mathrm{th}}$. (3) The temperature is lower than threshold temperature, $T_{\text {th }}$ (e.g., Navarro \& White 1993; Katz et al. 1996; Stinson et al. 2006). We adopt $n_{\text {th }}=100 \mathrm{~cm}^{-3}$ for our fiducial model, which is the mean density of giant molecular clouds (GMCs). We adopt $T_{\text {th }}=1000 \mathrm{~K}$ for our fiducial model. The value of $T_{\mathrm{th}}$ is insensitive to the final structure of galaxies (Saitoh et al. 2008).

When a gas particle satisfies the three conditions above, it becomes eligible to form new collisionless star particles. Star particles are produced by the following probability according to the prescription of Katz (1992); Katz et al. (1996):

$$
p=\frac{m_{\mathrm{gas}}}{m_{\star}}\left\{1-\exp \left(-c_{\star} \frac{d t}{t_{\mathrm{dyn}}}\right)\right\},
$$

where $m_{\star}$ and $m_{\text {gas }}$ are the mass of star and gas particles, respectively, and $c_{\star}$ is the dimensionless star formation efficiency (SFE) parameter. We set $m_{\star}=m_{\text {gas }} / 3$ following Okamoto et al. (2003, 2005). The mass of a gas particle of our fiducial model is initially assumed as $4.0 \times 10^{2} M_{\odot}$, while it is reduced by star formation. When the mass of a gas particle becomes lower than one-third of initial mass, the particle is converted into a collisionless particle. The dimensionless SFE parameter of our fiducial model $\left(c_{\star}=0.033\right)$ is chosen based on the slow star-formation model (Zuckerman \& Evans 1974; Krumholz \& Tan 2007). Saitoh et al. (2008) suggest that when $n_{\mathrm{th}}=100 \mathrm{~cm}^{-3}$ is adopted, the final results are fairly insensitive to the adopted value of $c_{\star}$ in their MW model. In the Appendix, we also confirm this result in our dSph models.

Each star particle is treated as a single stellar population (SSP), i.e. each star particle is assumed to be an assembly of stars with the same age and the same metallicity. The initial mass function (IMF) of star particles is the Salpeter IMF: $\phi \equiv m^{-x}$, where $x=1.35$ (Salpeter 1955) with mass range of $0.1-100 M_{\odot}$. We set the progenitor mass of CCSNe to be $8-40 M_{\odot}$. In this model, stars more massive than $40 M_{\odot}$ end their lives as black holes. Star particles, which explode in a time interval of $\Delta t$ are selected by the following probability $\left(p_{\mathrm{CCSNe}}\right)$,

$$
p_{\mathrm{CCSNe}}=\frac{\int_{m(t)}^{m(t+\Delta t)} \phi\left(m^{\prime}\right) m^{\prime-1} \mathrm{~d} m^{\prime}}{\int_{m(t)}^{8 M_{\odot}} \phi\left(m^{\prime}\right) m^{\prime-1} \mathrm{~d} m^{\prime}},
$$

where $m(t)$ is the turn off mass at age $t$. Each CCSN explosion distributes thermal energy of $10^{51} \mathrm{erg}$ to the surrounding SPH particles. The mass of one star particle is $\sim 100 M_{\odot}$. When a particle explode as CCSNe, the number of CCSNe inside each star particle corresponds to $\sim 1$. This method is also adopted in other studies (e.g., Okamoto et al. 2008; Saitoh et al. 2008). In addition to the SN feedback, we implement heating by $\mathrm{H}_{\mathrm{II}}$ region formed around young stars. The number of the Ly $\alpha$ photons is evaluated using PÉGASE (Fioc \& Rocca-Volmerange 1997). The parameters of these baryonic physics are listed in Table 1

\subsection{Chemical enrichment process}

We take into account both CCSNe and NSMs in our models. We set initial gas metallicity equals to zero. CCSNe produce $\mathrm{Fe}$ and NSMs produce $\mathrm{Eu}$, which is regarded as a representative element of $r$-process. Binary black hole-neutron star mergers are also expected to eject $r$-process elements (e.g., Korobkin et al. 2012; Mennekens \& Vanbeveren 2014). However, they affect the rate of production of $r$-process elements by several factors which are much smaller than the uncertainty of the rate of NSMs. We therefore only implement NSMs for simplicity. We assume that gas particles around a star particle are enriched with metals when a CCSN or NSM occurs in a star particle. Metals are distributed in 32 nearest neighbor particles using weights of SPH kernel. Mass of element $\mathrm{X}$ in the $j$ th neighbor particle ejected by $i$ th star particle, $\Delta M_{\mathrm{X}, j}$, is given by

$$
\Delta M_{\mathrm{X}, j}=\frac{m_{j}}{\rho_{i}} M_{\mathrm{X}, i} W\left(r_{i j}, h_{i j}\right),
$$

where $r_{i j}$ is the distance between particle $i$ and $j, h_{i j}$ is the smoothing length, and $W\left(r_{i j}, h_{i j}\right)$ is the SPH kernel given by a cubic spline function (e.g., Kawata et al. 2001) and the density of the gas particles is given as

$$
\rho_{i}=\sum_{i \neq j} m_{j} W\left(r_{i j}, h_{i j}\right)
$$

Smoothing length is the scale of containing $N_{\text {ngb }}$ nearest neighbor particles. Following Saitoh et al. (2008), we set $N_{\text {ngb }}=32 \pm 2$ as a fiducial value.

The iron yield of CCSNe are taken from Nomoto et al. (2006). The scope of this paper is to discuss the abundance ratio of $r$-process elements in EMP stars before type Ia supernovae (SNe Ia) contribute. We do not implement SNe Ia in our simulation.

The NSM rate and the merger time $\left(t_{\mathrm{NSM}}\right)$ are highly uncertain. We therefore vary them $\sim 2$ dex in our simulations. We regard a number fraction of NSMs to the total number of neutron stars, $f_{\mathrm{NSM}}$, as a parameter, which determines the NSM rate. In this model, we assume the mass range of NS progenitor mass as 8-20 $M_{\odot}$. We set the upper mass of NSM progenitor stars as 20 $M_{\odot}$ from the lower limit of the mass of a black hole formation (Dominik et al. 2012). We set $f_{\mathrm{NSM}}=0.01$ as a fiducial value. The corresponding NSM rate in a MilkyWay size galaxy is $\sim 10^{-4} \mathrm{yr}^{-1}$. It is within the values of the Galactic disk $\sim 10^{-6}-10^{-3} \mathrm{yr}^{-1}$, estimated from observed compact binaries (Abadie et al. 2010a).

Yields of NSMs are related to the rate of NSMs. $[\mathrm{Eu} / \mathrm{Fe}]$ at $[\mathrm{Fe} / \mathrm{H}]=0$ is expected to be $\sim 0.5$ without $\mathrm{SNe}$ Ia because solar $\mathrm{Fe}$ is estimated to be produced $\sim 60-65 \%$ by SNe Ia, and $\sim 35-40 \%$ by CCSNe 
TABLE 1

PARAMETERS OF BARYON PHYSICS.

\begin{tabular}{|c|c|c|c|}
\hline Quantity & Symbol & Fiducial values $^{\mathrm{a}}$ & Variation \\
\hline Dimensionless SFE parameter & $c_{\star}$ & 0.033 & $0.033,0.5$ \\
\hline Threshold density for star formation & $n_{\text {th }}$ & $100 \mathrm{~cm}^{-3}$ & $0.1-100 \mathrm{~cm}^{-3}$ \\
\hline Threshold temperature for star formation & $T_{\text {th }}$ & $1 \times 10^{3} \mathrm{~K}$ & $1 \times 10^{3}-3 \times 10^{4} \mathrm{~K}$ \\
\hline SN explosion energy & $\epsilon_{\mathrm{SN}}$ & $1 \times 10^{51}$ erg & $(0.03-1) \times 10^{51} \mathrm{erg}$ \\
\hline
\end{tabular}

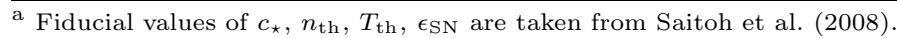

(e.g., Goswami \& Prantzos 2000; Prantzos 2008). We thus simply set the yield of $r$-process elements to be $[\mathrm{Eu} / \mathrm{Fe}]=0.5$ at $[\mathrm{Fe} / \mathrm{H}]=0$.

Observed $r$-process elemental abundance ratio such as $[\mathrm{Eu} / \mathrm{Fe}]$ indicates that the production of $r$-process elements should have occurred before SNe Ia start to contribute $(>1$ Gyr) to galactic chemical evolution (Maoz et al. 2012). Minimum merger time of NSMs needs to be shorter than the typical delay time of SNe Ia. As already mentioned, the most plausible merger time of NSMs is regarded as $\sim 100$ Myr (e.g., Lorimer 2008; Dominik et al. 2012). We thus set the merger time of NSMs is 100 Myr as a fiducial value.

\subsection{Definition of abundance of newly formed stars}

The abundance of a star must be identical to the abundance of the gas, which formed the star. The abundance of a newly formed star particle inherits (1) the abundance of the star-forming gas particle (e.g., Raiteri et al. 1999; van de Voort et al. 2015; Shen et al. 2015); (2) the abundance of the average of gas particles within a SPH kernel (e.g., Steinmetz \& Müller 1994; Kobayashi \& Nakasato 2011; Shen et al. 2015). Method (1) does not have a metal mixing process except for hydrodynamical mixing process such as stellar winds, outflows and inflows due to the SN explosion. [Eu/Fe] produced in method (1) is discussed in 4.1 . On the other hand, in method (2), a metal mixing process is taken into account. We use a metallicity averaged over 32 neighbor gas particles in a SPH kernel to a newly born star particle. The region can be regarded as star-forming region, which corresponds to $\sim 10^{4} M_{\odot}$. This mass corresponds to the typical size of giant molecular clouds (GMCs) (e.g., Larson 1981; Liszt et al. 1981; Sanders et al. 1985; Solomon et al. 1987; Harris \& Pudritz 1994; Hever et al. 2009). We discuss results inferred from method (2) in 4.2

Massive stars tend to be born in clusters and associations (Lada \& Lada 2003). Clusters and OB associations form from GMCs. Observations of stars in open clusters suggest that their metallicity is homogeneous (De Silva et al. 2007a, b; Pancino et al. 2010; Bubar \& King 2010; De Silva et al. 2011: Ting et al. 2012; Reddy et al. 2012; De Silva et al. 2013; Reddy et al. 2013). Feng \& Krumholz (2014) theoretically show that turbulent mixing in star-forming regions causes this homogeneity. The timescale of metal mixing is determined by the local dynamical time of star-forming regions ( $\lesssim 1 \mathrm{Myr}$ ). This timescale is much shorter than the typical timescale of star formation $(\gtrsim 10$ Myr) in slow star formation model (Zuckerman \& Evans 1974; Krumholz \& Tan 2007). We thus assume that the metals are instantaneously mixed in star-forming re- gions. We discuss the effect of different implementation on abundance ratios in galaxies in 4.1 and 4.2

\subsection{Models of dSphs}

We follow initial conditions of dSph models adopted in Revaz et al. (2009) and Revaz \& Jablonka (2012). We assume the density profile of dark matter, $\rho$, as follows:

$$
\rho=\frac{\rho_{c}}{1+\left(r / r_{c}\right)^{2}},
$$

where $\rho_{c}$ is the central density and $r_{c}$ is the core radius. Gas particles are also distributed along with the profile. We set $r_{\mathrm{c}}=1.0 \mathrm{kpc}, r_{\max }=7.1 \mathrm{kpc}$, and $M_{\mathrm{tot}}=7$ $\times 10^{8} M_{\odot}$, according to Revaz \& Jablonka (2012). We adopt 0.15 of mass ratio of gas to dark matter particles (baryon fraction, $f_{\mathrm{b}} \equiv \Omega_{\mathrm{b}} / \Omega_{\mathrm{m}}$ ). The value of $f_{\mathrm{b}}$ is taken from Planck Collaboration (2014).

Following Revaz et al. (2009), we assume an isotropic velocity dispersion of dark matter particles, $\sigma(r)$, for spherical distribution (Hernquist 1993; Binney \& Tremaine 2008),

$$
\sigma^{2}(r)=\frac{1}{\rho(r)} \int_{r}^{\infty} \mathrm{d} r^{\prime} \rho\left(r^{\prime}\right) \frac{\partial \Phi\left(r^{\prime}\right)}{\partial r^{\prime}},
$$

where $\Phi(r)$ is the gravitational potential. For gas particles, we set a velocity equal to zero and an initial temperature of $10^{4} \mathrm{~K}$. For both dark matter and gas particles, we adopt gravitational softening length $\left(\epsilon_{\mathrm{g}}\right)$ of $28 \mathrm{pc}$ for runs with the initial total number $(N)$ of $2^{14}, \epsilon_{\mathrm{g}}=14 \mathrm{pc}$ for runs with $N=2^{16}, 2^{17}$, and $2^{18}$, and $\epsilon_{\mathrm{g}}=7 \mathrm{pc}$ for runs with $N=2^{19}$. We run our simulations over 14 Gyr. The parameters of our model galaxies are listed in Table 2. Table 3 summarizes all runs discussed in this paper.

\section{CHEMO-DYNAMICAL EVOLUTION OF DWARF SPHEROIDAL GALAXIES}

\subsection{Dynamical evolution of $d S p h$ models}

We discuss chemo-dynamical evolution of model s000 to confirm that the parameter set of this model is appropriate for the case of dSphs. Parameter dependence is discussed in the Appendix.

Figure 1 shows the evolution of the spatial distribution of gas and stars of model s000. Upper panels of Figure 11 show the gas density maps of model s000 at 0 Gyr, 1 Gyr, 5 Gyr, and 10 Gyr from the beginning of the simulation. The gas monotonically collapses during the first 1 Gyr. Then, gas density reduces by star formation and gas outflow by energy feedback of SNe. Red colored area in upper panels of Figure 1 corresponds to the star-forming region where the number density of gas 
TABLE 2

Parameters of the initial CONDition.

\begin{tabular}{lll}
\hline \hline \multicolumn{1}{c}{ Quantity } & Symbol & \multicolumn{1}{c}{ Values $^{\mathrm{a}}$} \\
\hline Total mass & $M_{\text {tot }}$ & $7 \times 10^{8} M_{\odot}$ \\
Core radius & $r_{\mathrm{c}}$ & $1 \mathrm{kpc}$ \\
Initial outer radius & $r_{\max }$ & $7.1 \mathrm{kpc}$ \\
Baryon fraction & $f_{\mathrm{b}}$ & 0.15 \\
\hline a Values are &
\end{tabular}

Revaz \& Jablonka (2012).

TABLE 3

LIST OF MODELS.

\begin{tabular}{lcrrrrrrrrrr}
\hline \hline Model & $N$ & $\begin{array}{c}m_{\mathrm{DM}} \\
\left(10^{3} M_{\odot}\right)\end{array}$ & $\begin{array}{c}m_{\mathrm{gas}} \\
\left(10^{3} M_{\odot}\right)\end{array}$ & $\begin{array}{c}\epsilon_{\mathrm{g}} \\
(\mathrm{pc})\end{array}$ & $\begin{array}{c}n_{\mathrm{th}} \\
\left(\mathrm{cm}^{-3}\right)\end{array}$ & $\begin{array}{c}T_{\mathrm{th}} \\
\left(10^{3} \mathrm{~K}\right)\end{array}$ & $\begin{array}{c}\epsilon_{\mathrm{SN}} \\
\left(10^{51} \mathrm{erg}\right)\end{array}$ & $\mathrm{N}_{\mathrm{ngb}}$ & Mixing & $\begin{array}{c}t_{\mathrm{NSM}} \\
(\mathrm{Myr})\end{array}$ & $f_{\mathrm{NSM}}$ \\
\hline $\mathrm{s} 000$ & $2^{19}$ & 2.3 & 0.4 & 7 & 100 & 1 & 1 & 32 & no & 100 & 0.01 \\
\hline $\mathrm{m} 000$ & $2^{19}$ & 2.3 & 0.4 & 7 & 100 & 1 & 1 & 32 & yes & 100 & 0.01 \\
$\mathrm{mN16}$ & $2^{19}$ & 2.3 & 0.4 & 7 & 100 & 1 & 1 & 16 & yes & 100 & 0.01 \\
$\mathrm{mN64}$ & $2^{19}$ & 2.3 & 0.4 & 7 & 100 & 1 & 1 & 64 & yes & 100 & 0.01 \\
$\mathrm{~m} 014$ & $2^{14}$ & 72.6 & 12.8 & 28 & 100 & 1 & 1 & 32 & yes & 100 & 0.01 \\
$\mathrm{~m} 016$ & $2^{16}$ & 18.2 & 3.2 & 14 & 100 & 1 & 1 & 32 & yes & 100 & 0.01 \\
$\mathrm{~m} 017$ & $2^{17}$ & 9.1 & 1.6 & 14 & 100 & 1 & 1 & 32 & yes & 100 & 0.01 \\
$\mathrm{~m} 018$ & $2^{18}$ & 4.5 & 0.8 & 14 & 100 & 1 & 1 & 32 & yes & 100 & 0.01 \\
\hline $\mathrm{mExt}$ & $2^{19}$ & 2.3 & 0.4 & 7 & 0.1 & 30 & 0.03 & 32 & yes & 100 & 0.01 \\
\hline $\mathrm{m} 10$ & $2^{19}$ & 2.3 & 0.4 & 7 & 100 & 1 & 1 & 32 & yes & 10 & 0.01 \\
$\mathrm{mt500}$ & $2^{19}$ & 2.3 & 0.4 & 7 & 100 & 1 & 1 & 32 & yes & 500 & 0.01 \\
\hline mr0.001 & $2^{19}$ & 2.3 & 0.4 & 7 & 100 & 1 & 1 & 32 & yes & 100 & 0.001 \\
mr0.1 & $2^{19}$ & 2.3 & 0.4 & 7 & 100 & 1 & 1 & 32 & yes & 100 & 0.1 \\
\hline
\end{tabular}

Note. — Parameters adopted in our models: (1)Model: Name of our models. Models named "000" adopt the fiducial parameter set. Model s000 is discussed in $\$ 3$ and 4.1 Models m000 to m018 are discussed in 4.2 Model mExt is discussed in 4.3 Models mt10 and mt500 are discussed in 44.4 Models mr0.001 and mr0.1 are discussed in 44.5 (2) $N$ : Initial total number of particles. (3) $m_{\mathrm{DM}}$ : Mass of one dark matter particle. (4) $m_{\text {gas }}$ : Initial mass of one gas particle. (5) $\epsilon_{\mathrm{g}}$ : Gravitational softening length. (6) $n_{\mathrm{th}}$ : Threshold density for star formation. (7) $T_{\mathrm{th}}$ : Threshold temperature for star formation. (8) $e_{\mathrm{SN}}$ : SN feedback energy. (9) $N_{\mathrm{ngb}}$ : Number of nearest neighbor particles. (10) Mixing: With (yes) or without (no) metal mixing in star-forming region. (11) $t_{\mathrm{NSM}}$ : Merger time of NSMs. (12) $f_{\mathrm{NSM}}$ : Fraction of NSMs.

is larger than $100 \mathrm{~cm}^{-3}$. As shown in this figure, starforming region is strongly confined at the center of the galaxy. The red area is largest at 1 Gyr. This indicates that star formation is most active after $\sim 1$ Gyr from the beginning of the simulation.

Lower panels of Figure 1 show stellar density maps at 0 Gyr, 1 Gyr, 5 Gyr, and 10 Gyr. The distribution of stars at $1 \mathrm{Gyr}$ is associated with the high-density region of gas (red region in Figure 1). As shown in these figures, stars continuously form in the inner region of this model galaxy for over $10 \mathrm{Gyr}$ and stellar density distribution expands with time from the center to the outer region. At 10 Gyr, the morphology of the galaxy becomes spherical symmetry.

In order to quantitatively discuss the structural and dynamical properties of models, we investigate the radial profiles. Figure 2 shows the radial profiles of model s000. We define the galactic center using potential minimum. The values in each point are calculated in each bin from the center to the outer region.

Figure 2 (a) shows time variation of the dark matter density profile. At 1 Gyr, the dark matter follows the initial density profile given in Eq. (5). After the collapse in the first $1 \mathrm{Gyr}$, the shape of the dark matter profile does not change over 10 Gyr.

Figure 2(b) shows time variation of the gas density profile. Inner region $(\lesssim 1 \mathrm{kpc})$ of the gas density profile follows the evolution of the dark matter density profile. Outer region of gas is blown away due to the outflow induced by SNe. In addition, the total amount of gas reduces because of star formation. However, gas still remains even at 10 Gyr. As in the Appendix, all of our models have gas at 10 Gyr. The observed LG dSphs in contrast have no or little gas (e.g. McConnachie 2012). This result suggests that physical processes such as ram pressure and tidal stripping are required to remove all gas away from dSphs (Mayer et al. 2006; Nichols et al. 2014).

As shown in Figure 2 (c), the stellar density profile of our simulation well reproduces observations. In Figure 2 (c), we present the stellar density profiles. Stars distribute within $\sim 1 \mathrm{kpc}$, which is consistent with the observed tidal radii $(\sim 0.5-3 \mathrm{kpc})$ of $\mathrm{dSphs}$ in the $\mathrm{LG}$ (Irwin \& Hatzidimitriou 1995). The density profile of stars is basically associated with the dark matter density profile.

Figure 2 (d) shows the stellar velocity dispersion profile. The observed stellar velocity dispersion of dSphs is almost constant within $\sim 1 \mathrm{kpc}$ from the center (Walker et al. 2009). Model s000 has similar properties with the observed radial stellar velocity dispersion profiles inside $1 \mathrm{kpc}$ from the center in the LG dSphs.

\subsection{Time variations of the star formation rate}




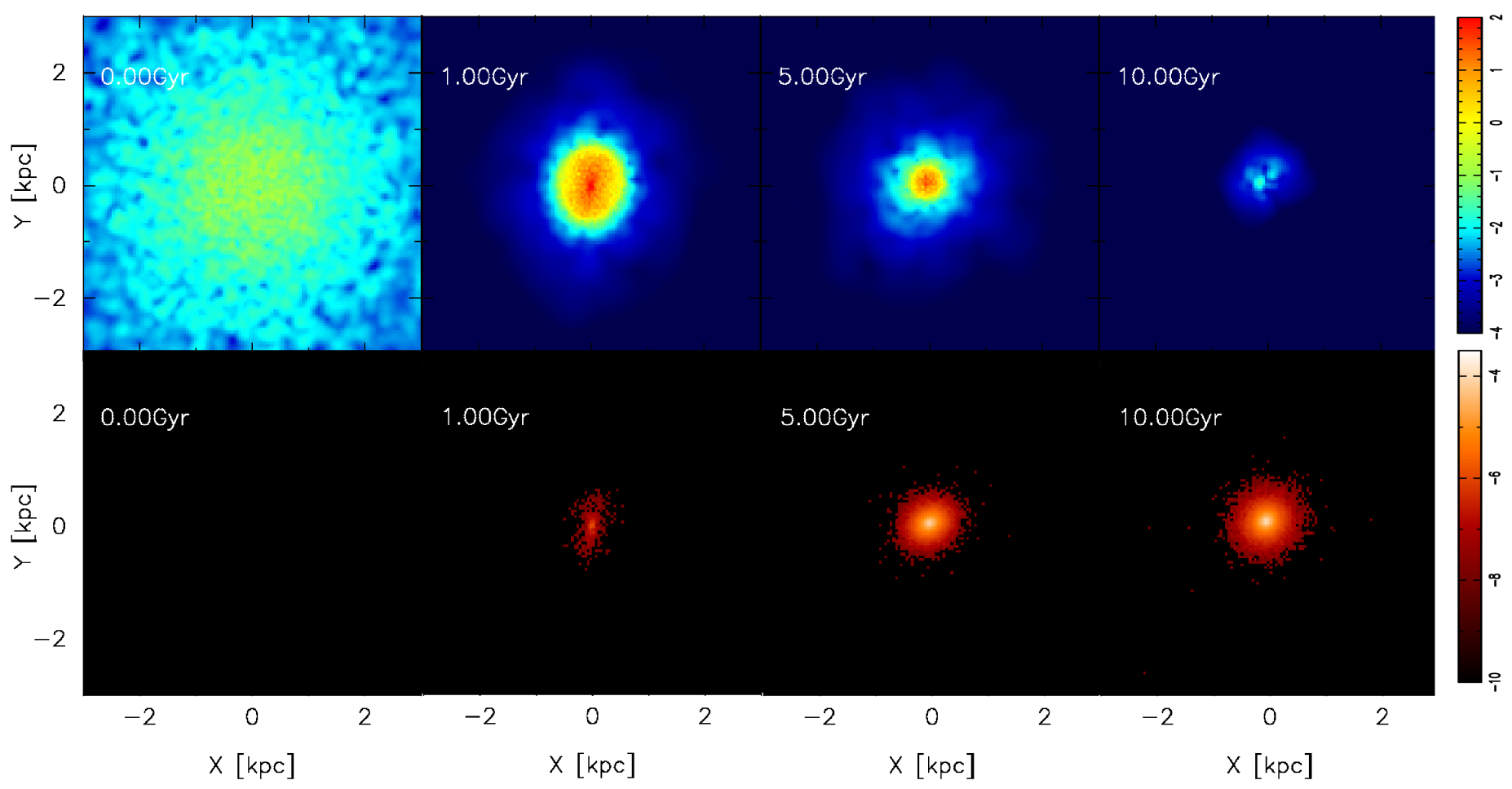

FIG. 1. - Evolution of model s000. Upper panels: snapshots of slice gas density in log scale, between $10^{-4} \mathrm{~cm}^{-3}(\mathrm{blue})$ and $10^{2} \mathrm{~cm}^{-3}$ (red). Lower panels: snapshots of stellar surface density with $\log$ scale, between $10^{-10} 10^{10} M_{\odot} \mathrm{kpc}^{-2}(\mathrm{black})$ and $10^{-3.5} 10^{10} M_{\odot} \mathrm{kpc}^{-2}$ (white).
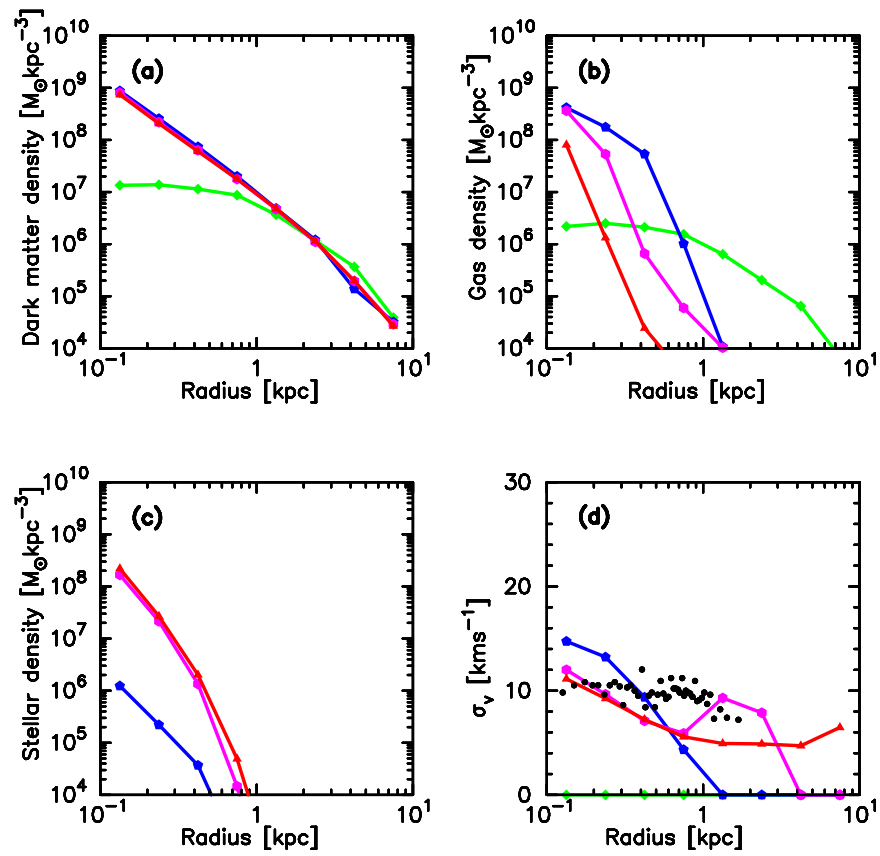

Fig. 2.- Radial profiles of model s000 at $t=0$ Gyr (green), 1 Gyr (blue), 5 Gyr (magenta), and 10 Gyr (red). (a): radial dark matter density profile. (b): radial gas density profile. (c): radial stellar density profile. (d): radial stellar velocity dispersion profile. Black dots are observed stellar velocity dispersion in the Fornax dSph (Walker et al. 2009).

Figure 3 shows the time variation of the SFR in model s000 (red curve) and the observed values of the Fornax and the Sculptor dSphs (de Boer et al. 2012a, b). The
SFR of model s000 is peaked at $\sim 2$ Gyr. Gas density increases with accretion (see Figure 2 (b)) and finally reaches the threshold density for star formation. On the other hand, SN feedback drives gas away from the inner region to the outer region (Hopkins et al. 2011). Because of the shallow gravitational potential and high threshold density for star formation $\left(n_{\mathrm{th}}=100 \mathrm{~cm}^{-3}\right)$, SN feedback significantly affects the timescale of gas accretion. It therefore takes long time $(\sim 1 \mathrm{Gyr})$ to reach the peak of the SFR. The SFR of model s000 $\left(\sim 10^{-3} M_{\odot} \mathrm{yr}^{-1}\right)$ is consistent with the observed value of the Fornax and the Sculptor dSphs inferred from color-magnitude diagram analysis $\left(\sim 10^{-3} M_{\odot} \mathrm{yr}^{-1}\right.$, de Boer et al. 2012a b). The SFR of model mExt (magenta curve) is discussed in 4.3 .

\subsection{Metallicity distribution}

Metallicity distribution is one of the best properties to test reliability of chemical evolution models. Figure 4 compares metallicity distribution between model s000 and observation. All data of the Fornax (Kirby et al. 2010) and the Sculptor dSphs (Kirby et al. 2009, 2010; Kirby \& Cohen 2012). Metallicity distribution of model s000 is almost consistent with the observed value of the Sculptor dSph. The metallicity at the peak of the distribution of model $\mathrm{s} 000$ is $[\mathrm{Fe} / \mathrm{H}]=-1.57$, which is lower than that of the Fornax dSph, $[\mathrm{Fe} / \mathrm{H}]=-1.06$ (Kirby et al. 2013). This is because we do not implement SNe Ia in this model, while the Fornax dSph must be significantly affected by the metal ejection of SNe Ia (e.g., Kirby et al. 2010). If we take account of the products of SNe Ia, the peak metallicity is expected to shift by $\sim 0.5$ dex to the higher metallicity, which is closer 


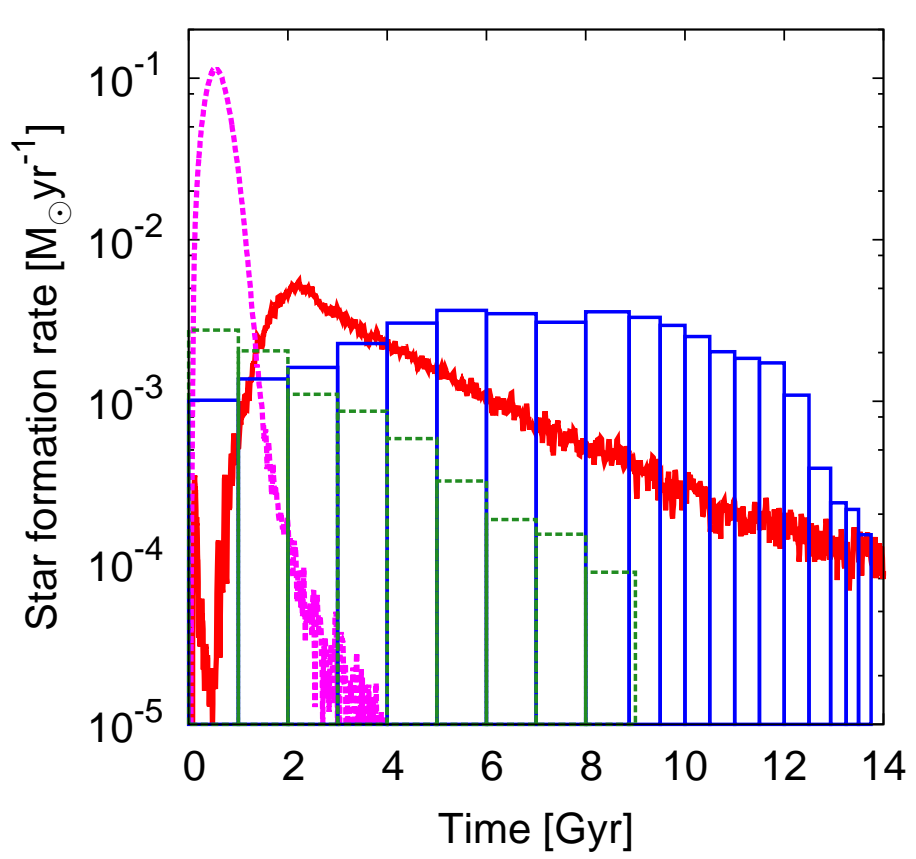

FIG. 3.- The SFR as a function of time for our models. Red and magenta-dashed curves represent the SFR of models s000 and mExt, respectively. Blue and green-dashed histograms represent the observed SFR of the Fornax dSph (de Boer et al. 2012b) and the Sculptor dSph (de Boer et al. 2012a), respectively.

value to that of the Fornax dSph.

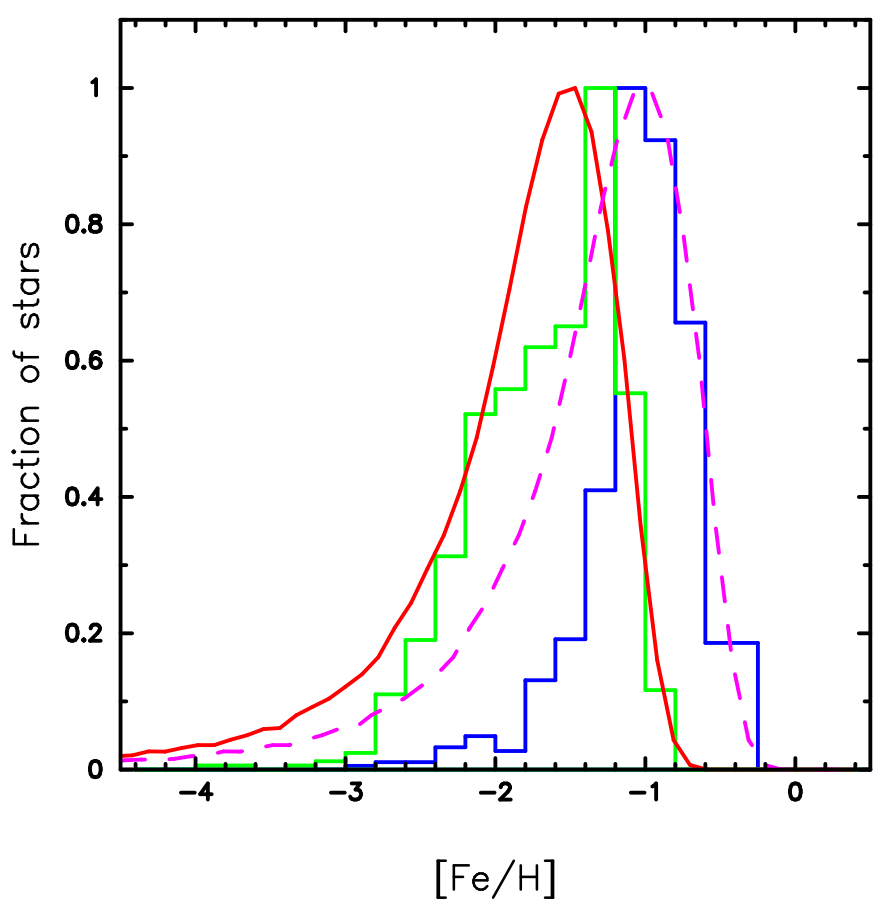

FIG. 4.- Metallicity distribution of model s000 (red curve), model s000 but $[\mathrm{Fe} / \mathrm{H}]$ is shifted to 0.5 dex taking into account the effect of SNe Ia (magenta-dashed curve), the observed value of the Fornax dSph (blue histogram) (Kirby et al.2010), and the Sculptor dSph (green histogram) (Kirby et al. 2009, 2010; Kirby \& Cohen 2012).

\section{ENRICHMENT OF $R$-PROCESS ELEMENTS IN DWARF SPHEROIDAL GALAXIES}

\subsection{Enrichment of $r$-process elements without metal mixing in star-forming region}

In this section, we discuss $[\mathrm{Eu} / \mathrm{Fe}]$ as a function of $[\mathrm{Fe} / \mathrm{H}]$ predicted in model s000. In model s000, the metallicity of a star particle inherits that of the gas particle from which the star particle was formed, according to the method (1) in $\$ 2.3$ Figure 5 shows $[\mathrm{Eu} / \mathrm{Fe}]$ as a function of $[\mathrm{Fe} / \mathrm{H}]$ predicted in model s000. We also put the observed data of the Galactic halo and several dSphs, i.e., Carina, Draco, Leo I, Sculptor, and Ursa Minor dSphs (SAGA database, Suda et al. 2008, 2011, 2014; Yamada et al. 2013), excluding carbon-enhanced stars, which are possibly affected by gas transfer in binaries. We also exclude stars in the Fornax dSph because some of them have extremely high $[\mathrm{Eu} / \mathrm{Fe}](>$ $0.5 \mathrm{dex})$ due to significant contamination of $s$-process (Letarte et al. 2010). As shown in Figure 5] stars of highly $r$-process enhanced stars; $[\mathrm{Eu} / \mathrm{Fe}]>1$ (so-called $r$-II stars), are over-abundant. In addition, $r$-deficient stars in $-2<[\mathrm{Fe} / \mathrm{H}]<-1$ are predicted. Such low $[\mathrm{Eu} / \mathrm{Fe}]$ stars are not seen in the observation. These stars are not simply caused by delayed production of Eu by NSMs. In fact, the average value of $[\mathrm{Eu} / \mathrm{Fe}]$ does not increase with metallicity at around $[\mathrm{Fe} / \mathrm{H}] \sim-2$. The significant dispersions of chemical components among gas particles seem to be rather essential. The relations between the galactic age and abundances of Fe and Eu show the reason why such large dispersions are seen in low metallicity region.

Figure [6 (a) shows $[\mathrm{Fe} / \mathrm{H}]$ as a function of time. Metallicity obviously increases with time, as CCSNe produce iron. The metallicity however has more scatters at the earlier time, especially during the first few Gyrs. Then, later formed stars are enriched by more numerous CCSNe, and as a result, the dispersion of stellar metallicity decreases with time. We denote the stars, which are formed from the gas enriched only by a single CCSN by black circles in Figure 6 (a). Their metallicity widely distributes over $\sim 3$ dex. These stars concentrate only in $\lesssim 2$ Gyr.

Figure 6] (b) shows $[\mathrm{Eu} / \mathrm{H}]$ as a function of time. In contrast to Figure 6 (a), large star-to-star scatters in $[\mathrm{Eu} / \mathrm{H}]$ remain over the whole evolution of the galaxy. As shown in black circles in Figure 6 (b), gas particles affected by one NSM remain over 10 Gyr. One of the reasons must be the low rate of NSMs. The rate of NSMs is one hundred times lower than that of CCSNe in this model. The total number of NSMs may not be enough to converge the $[\mathrm{Eu} / \mathrm{H}]$ in this model.

In addition to the low NSM rate, the efficiency of gas mixing in this model seems to cause unnaturally large scatters in $[\mathrm{Eu} / \mathrm{H}]$. If a star particle contains products from a single NSM, the value of $[\mathrm{Eu} / \mathrm{H}]$ must be determined by the distance from the NSM, which enriched the star-forming gas particle. However, as shown in Figure [6 (b), dispersion of such stars drags longer than 5-10 Gyrs, which is much longer than the merger time of NSMs. It implies that gas particles never change the abundance of Eu, unless other NSMs enrich them again, though in actual, gas clouds are expected to interact with others. 


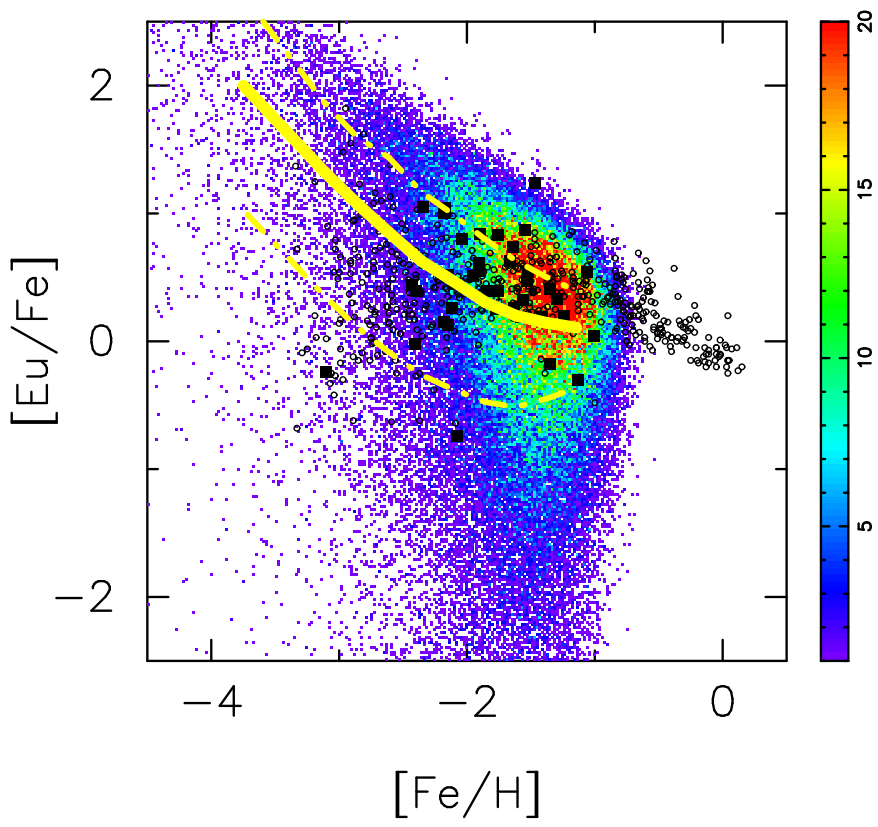

FIG. 5. - $[\mathrm{Eu} / \mathrm{Fe}]$ as a function of $[\mathrm{Fe} / \mathrm{H}]$ of model s000. Contour is the number of stars produced in our model, between 0 (purple) and 20 (red). Yellow curve is the median of model prediction. Dash-dotted curves are the first and third quartiles, respectively. Circles are the observed value of the Galactic halo stars (SAGA database, Suda et al. 2008, 2011; Yamada et al. 2013). Squares are the observed value of stars in Carina, Draco, Leo I, Sculptor, and Ursa Minor dSphs (SAGA database, Suda et al. 2014). Stars produced by our model are plotted within $0.5 \mathrm{kpc}$ from the center of our model galaxies.

In fact, observations of open clusters show that stellar metallicity is quite homogeneous in each cluster (e.g., De Silva et al. 2007a,b; Pancino et al. 2010; Bubar \& King 2010; De Silva et al. 2011; Ting et al. 2012; Reddy et al. 2012; De Silva et al. 2013; Reddy et al. 2013). The gas in star-forming region is possibly homogenized by hydro-dynamical effects, such as turbulent mixing (Feng \& Krumholz 2014).

In addition, previous studies suggest that the standard SPH simulations without metal mixing tend to predict a lower amount of gas with low metallicity and higher metallicity of inter galactic medium (Wiersma et al. 2009; Shen et al. 2010). Shen et al. (2015) suggest that it is difficult to reproduce the observed $[\mathrm{Eu} / \mathrm{Fe}]$ as a function of $[\mathrm{Fe} / \mathrm{H}]$ without metal mixing in star-forming region.

On the other hand, the fiducial model of van de Voort et al. (2015) reproduces the observed $[\mathrm{Eu} / \mathrm{Fe}]$ of metal-poor stars, although they adopt the same definition of metallicity as our model s000. They suggest that large-scale metal mixing such as galactic winds and hydrodynamical flows is important to reproduce the observed $[\mathrm{Eu} / \mathrm{Fe}]$ as a function of $[\mathrm{Fe} / \mathrm{H}]$. In their model, NSMs eject metals in the region of $3.5 \times 10^{6} M_{\odot}$, which is much larger than the swept-up mass of NSMs $\left(\sim 10^{4} M_{\odot}\right)$. The treatment of van de Voort et al. (2015) is identical to implement metal mixing.

\subsection{Effects of metal mixing on enrichment of $r$-process elements}

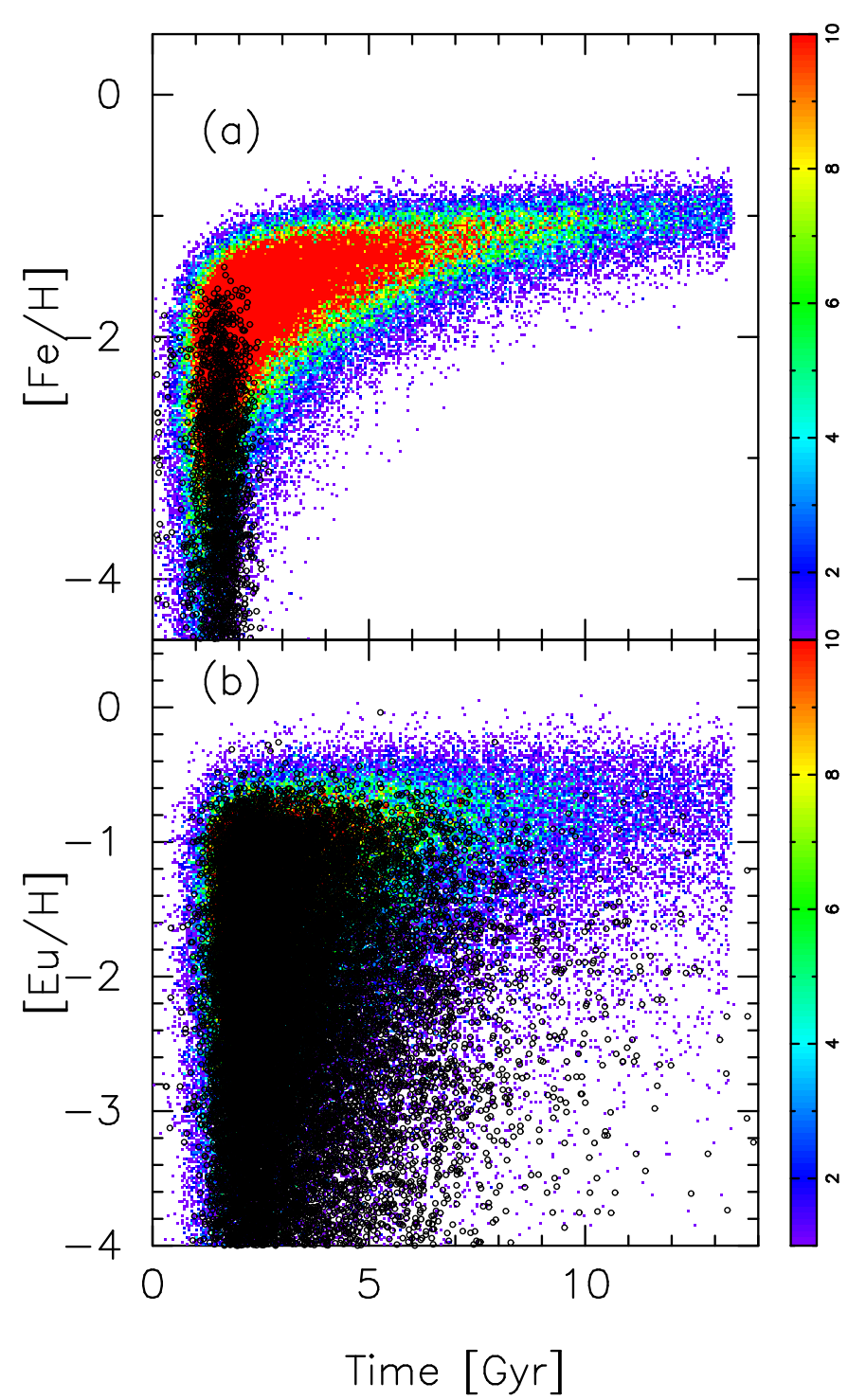

Fig. 6. - (a): $[\mathrm{Fe} / \mathrm{H}]$ as a function of time from the start of the simulation of model s000. (b): $[\mathrm{Eu} / \mathrm{H}]$ as a function of time from the start of the simulation of model s000. Black circles are stars formed from gas particles, which are affected by one CCSN. Contour is the same as Figure 5

As discussed in the previous section, the effect of mixing of enriched gas must be essential to account for the observed values of $[\mathrm{Eu} / \mathrm{Fe}]$ in metal-poor stars. Therefore, we take account of the effect of metal mixing according to the method (2) in $\$ 2.3$, and we adopt the average metallicity of gas particles in the SPH kernel of the progenitor gas particle for the metallicity of newly formed stars. Figure 7 shows $[\mathrm{Eu} / \mathrm{Fe}]$ as a function of $[\mathrm{Fe} / \mathrm{H}]$ with the metal mixing model, m000. Model m000 has the same parameter set for s000 except for the effect of metal mixing in star-forming region (see Table 3). As shown in this figure, $r$-deficient stars in $[\mathrm{Fe} / \mathrm{H}]$ of -2 to -1 seen in Figure 5 disappear due to metal mixing. The fraction of $r$-II stars is also reduced in this model due to adopted averaged metallicity in star-forming region. Model m000 apparently reproduces the observational tendency of $[\mathrm{Eu} / \mathrm{Fe}]$ in metal-poor stars much better than the model s000. Our model does not require the 
assumption of short merger time $\left(t_{\mathrm{NSM}} \lesssim 10 \mathrm{Myr}\right)$, which is required to reproduce observations in previous studies (e.g., Argast et al. 2004; Matteucci et al. 2014; Komiva et al. 2014; Tsujimoto \& Shigevama 2014).

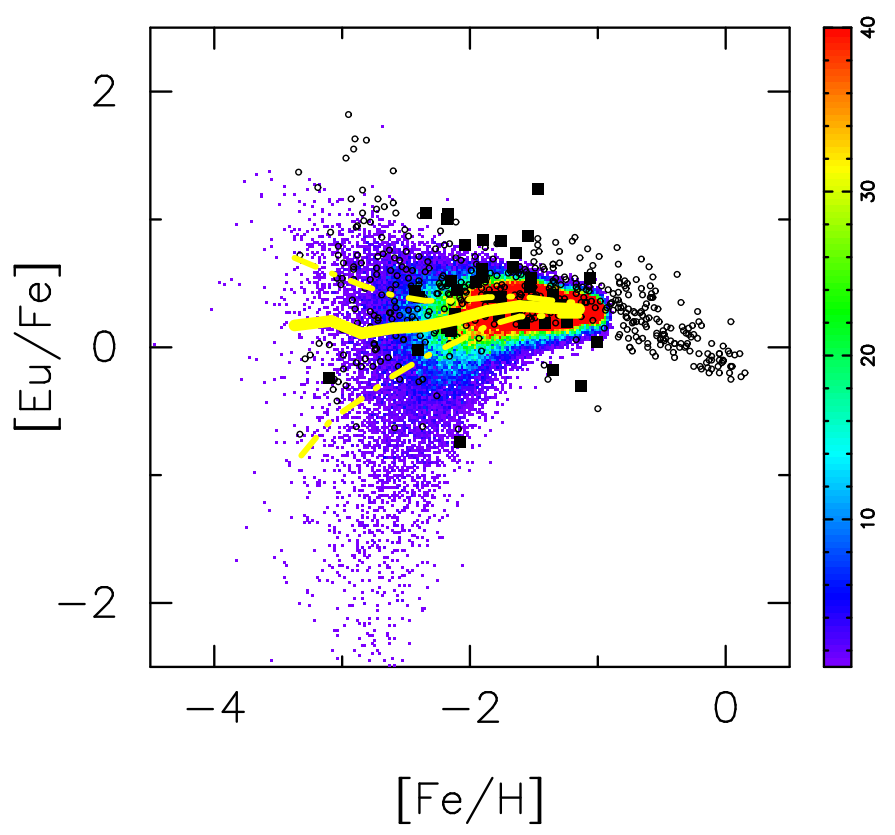

FIG. 7.- $[\mathrm{Eu} / \mathrm{Fe}]$ as a function of $[\mathrm{Fe} / \mathrm{H}]$ of the model m000. Symbols are the same as Figure 5

Figure 8 shows $[\mathrm{Eu} / \mathrm{Fe}]$ distributions in stars of $[\mathrm{Fe} / \mathrm{H}]$ $<-2.0$ predicted in s000 (without mixing model) and m000 (with mixing model). Observational values of the MW (red histogram) and dSphs (blue histogram) are provided by the SAGA database (Suda et al. 2008, 2011, 2014; Yamada et al. 2013). While model s000 overproduces stars with $[\mathrm{Eu} / \mathrm{Fe}]<-1$, model m000 significantly reduces the fraction of $r$-deficient stars. In addition, the fraction of $r$-II stars also reduces in model m000. This result therefore suggests that metal mixing in star-forming region is fairly important physical process to reproduce the observed $[\mathrm{Eu} / \mathrm{Fe}]$ as a function of $[\mathrm{Fe} / \mathrm{H}]$.

The predicted distribution of $[\mathrm{Eu} / \mathrm{Fe}]$ must be affected by the mass of the mixed gas $\left(M_{\text {mix }}\right)$, and the initial total number of particles $(N)$. We define $M_{\text {mix }}=N_{\text {ngb }} m_{\text {gas }}$. The mass of one gas particle $\left(m_{\text {gas }}\right)$ is proportional to $N^{-1}$. Table 4 lists the examined values of parameters and the corresponding mixing mass $\left(M_{\text {mix }}\right)$. Figure 9 (a) shows the median value and the dispersion of $[\mathrm{Eu} / \mathrm{Fe}]$ of models, which have different $N_{\text {ngb. }}$. The dispersion of $[\mathrm{Eu} / \mathrm{Fe}]$ becomes smaller with larger $N_{\text {ngb }}$ as shown in this figure. When we increase the $N_{\text {ngb }}$, the value of $M_{\text {mix }}$ increases and larger mass fraction of gas in the galaxy is mixed. Due to the effects of increasing $M_{\mathrm{mix}}$, the dispersion becomes smaller in the models with larger value of $N_{\text {ngb }}$.

Figure 9 (b) shows the median value of $[\mathrm{Eu} / \mathrm{Fe}]$ as a function of $[\mathrm{Fe} / \mathrm{H}]$ of models, which have different $N$. As shown in Figure 9 (b), the dispersion of [Eu/Fe] shows the similar tendency irrespective of $N$, i.e., it decreases with increasing of metallicity. When we adopt the larger value of $N$, more star particles are produced, i.e., the

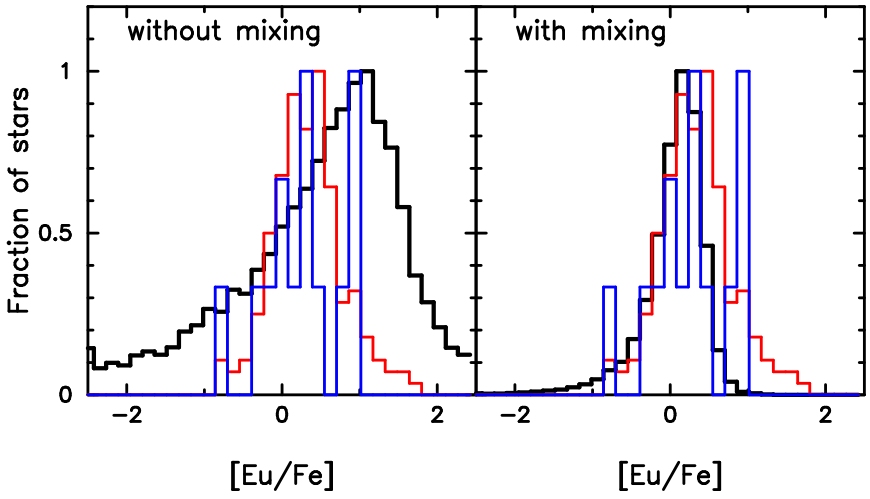

FIG. 8.- $[\mathrm{Eu} / \mathrm{Fe}]$ distribution of stars with $[\mathrm{Fe} / \mathrm{H}]<-2.0$ of our models (black histogram) and observation (red histogram: the Galactic halo stars, blue histogram: Carina, Draco, Leo I, Sculptor, and Ursa Minor dSphs). Data are compiled by SAGA database (Suda et al. 2008, 2011, 2014; Yamada et al. 2013). Left panel: plotted model is s000 (without metal mixing in star-forming region). Right panel: plotted model is m000 (with metal mixing in star-forming region).

TABLE 4

MASS OF METAL MIXING REGION.

\begin{tabular}{lrrr}
\hline \hline Model & $N$ & $N_{\text {ngb }}$ & $\begin{array}{c}M_{\text {mix }} \\
\left(10^{4} M_{\odot}\right)\end{array}$ \\
\hline m000 & $2^{19}$ & 32 & 1.3 \\
mN16 & $2^{19}$ & 16 & 0.6 \\
mN64 & $2^{19}$ & 64 & 2.7 \\
m018 & $2^{18}$ & 32 & 2.6 \\
m017 & $2^{17}$ & 32 & 5.1 \\
m016 & $2^{16}$ & 32 & 10.3 \\
m014 & $2^{14}$ & 32 & 41.0 \\
\hline \multicolumn{5}{c}{ Note. } & The columns corre- \\
spond to the name of model, initial \\
total number of particles $(N)$, the \\
number of nearest neighbor particles \\
$\left(N_{\text {ngb }}\right)$, and mass of the mixing re- \\
gion ( $\left.M_{\text {mix }}\right)$.
\end{tabular}

number of events of metal mixing increases. This means that metals are more mixed in the star-forming region if $M_{\text {mix }}$ is constant. However, $M_{\text {mix }}$ is defined to be proportional to $N^{-1}$. Models with larger value of $N$ have smaller value of $M_{\text {mix }}$. The effect of increasing $N$ offsets the effect made by decreasing $M_{\text {mix }}$. Thus, the dispersion is not affected by $N$.

\subsection{The relationship between the relative $r$-process abundance ratio and the star formation rate}

The value and scatters in the abundance ratio of $[\mathrm{Eu} / \mathrm{Fe}]$ must be affected by SFR, especially for metalpoor stars. In order to examine the effect of SFR, we discuss $[\mathrm{Eu} / \mathrm{Fe}]$ as a function of $[\mathrm{Fe} / \mathrm{H}]$ with the model mExt, in which extremely weak SN feedback energy $\left(\epsilon_{\mathrm{SN}}=3 \times 10^{49} \mathrm{erg}\right)$ and low threshold density for star formation $\left(n_{\mathrm{th}}=0.1 \mathrm{~cm}^{-3}\right)$ are assumed (Table 3). According to Appendix A.2, the weaker feedback model produces higher SFR and model with smaller value of $n_{\text {th }}$ shifts the peak of SFR to the earlier time. Since mExt has lower values of $\epsilon_{\mathrm{SN}}$ and $n_{\mathrm{th}}$, stars are more easily produced comparing to the model m000. The SFR of model mExt during the first 1 Gyr rises rapidly and 


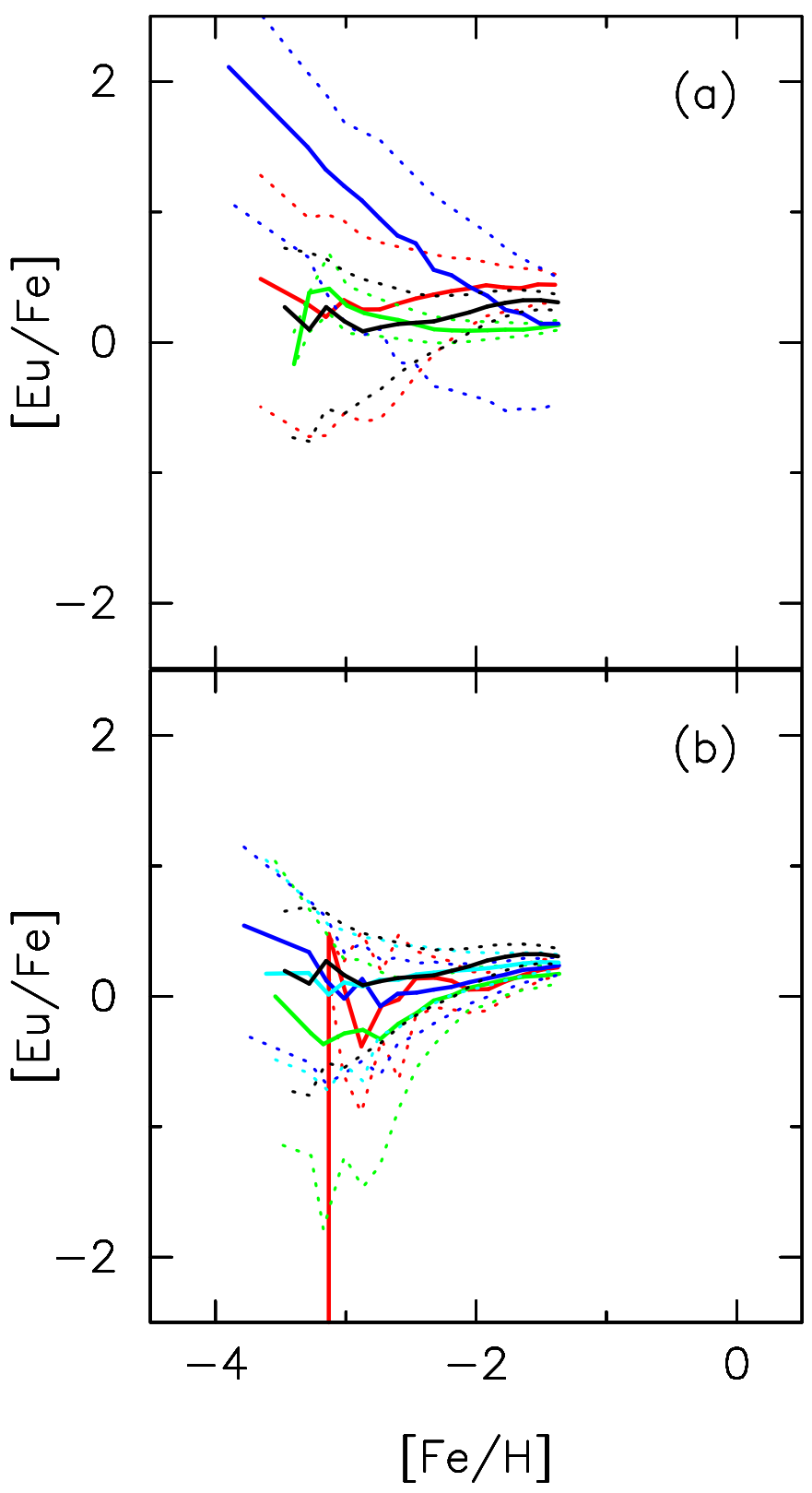

FIG. 9.- (a) Median $[\mathrm{Eu} / \mathrm{Fe}]$ as a function of $[\mathrm{Fe} / \mathrm{H}]$ produced by the different number of nearest neighbor particles $\left(N_{\mathrm{ngb}}\right)$. Solid curves are the median of model prediction and dashed curves are the first and third quartiles. Red curve represents mN16 $\left(N_{\text {ngb }}\right.$ $=16)$. Black curve represents m000 $\left(N_{\mathrm{ngb}}=32\right)$. Green curve represents $\mathrm{mN64}\left(N_{\mathrm{ngb}}=64\right)$. Blue curve represents s000 (without metal mixing in star-forming region). (b) Median $[\mathrm{Eu} / \mathrm{Fe}]$ as a function of $[\mathrm{Fe} / \mathrm{H}]$ produced by different initial number of particles $(N)$. Red curve represents m014 $\left(N=2^{14}\right)$. Green curve represents $\operatorname{m016}\left(N=2^{16}\right)$. Blue curve represents m017 $\left(N=2^{17}\right)$. Sky blue curve represents m018 $\left(N=2^{18}\right)$. Black curve represents $\operatorname{m000}\left(N=2^{19}\right)$.

is larger than $10^{-2} M_{\odot} \mathrm{yr}^{-1}$. The peak of SFR reaches $\sim 10^{-1} M_{\odot \mathrm{yr}^{-1}}$, while that of $\mathrm{m000}$ is $\lesssim 10^{-3} M_{\odot \mathrm{yr}^{-1}}$. The SFR of model mExt at $\lesssim 1$ Gyr is much higher than the observational values (Figure 3).

Figure [10 shows $[\mathrm{Eu} / \mathrm{Fe}]$ as a function of $[\mathrm{Fe} / \mathrm{H}]$ in model mExt. This model predicts significantly different $[\mathrm{Eu} / \mathrm{Fe}]$ from m000 (Figure 7) although both models adopt the same initial distribution of gas particles.
Model mExt produces a distribution of $r$-deficient stars around $[\mathrm{Fe} / \mathrm{H}] \sim-2$. In addition, no stars are produced below $[\mathrm{Fe} / \mathrm{H}] \sim-3$.

This difference is related to the timescale of chemical evolution in the early phase of the galaxy formation. For model m000, the median metallicity of gas particles at $1 \mathrm{Gyr}$ is $[\mathrm{Fe} / \mathrm{H}]=-3.32$. On the other hand, the median metallicity of gas particles in mExt is $[\mathrm{Fe} / \mathrm{H}]=-0.91$ at $1 \mathrm{Gyr}$. The SFE of model mExt is estimated to be $\sim 0.1-1 \mathrm{Gyr}^{-1}$. The value of $\mathrm{SFE}$ is comparable to some other inhomogeneous chemical evolution studies (Argast et al. 2004; Cescutti et al. 2015; Wehmeyer et al. 2015). Model mExt proceeds chemical evolution much faster than m000 due to the high SFR of mExt $\left(\sim 10^{-2} M_{\odot} \mathrm{yr}^{-1}\right)$. The $[\mathrm{Eu} / \mathrm{Fe}]$ as a function of $[\mathrm{Fe} / \mathrm{H}]$ in model mExt is inconsistent with the observation due to fast chemical evolution by the high SFR. This result suggests that the SFR and SFE in the early phase of dSphs are $\lesssim 10^{-3} M_{\odot} \mathrm{yr}^{-1}$ and $\lesssim 0.10$ $\mathrm{Gyr}^{-1}$, respectively, if the $r$-process elements are ejected by NSMs with a long merger time ( $100 \mathrm{Myr})$. As shown in Figure 3 the SFR of $\sim 10^{-3} M_{\odot} \mathrm{yr}^{-1}$ is a reasonable agreement with the observed value of the Fornax dSph (de Boer et al. 2012b). This SFR is also consistent with sub-halo models of Ishimaru et al. (2015) (Case 1 in their model suggests that the appropriate value of star formation efficiency for a sub-halo with a stellar mass of $10^{7} M_{\odot}$ should be $0.10 \mathrm{Gyr}^{-1}$, which corresponds to the order of SFR as $\left.\sim 10^{-3} M_{\odot} \mathrm{yr}^{-1}\right)$. They also suggest that the observed $[\mathrm{Eu} / \mathrm{Fe}]$ scatter in metal-poor stars by NSMs with a long merger time.

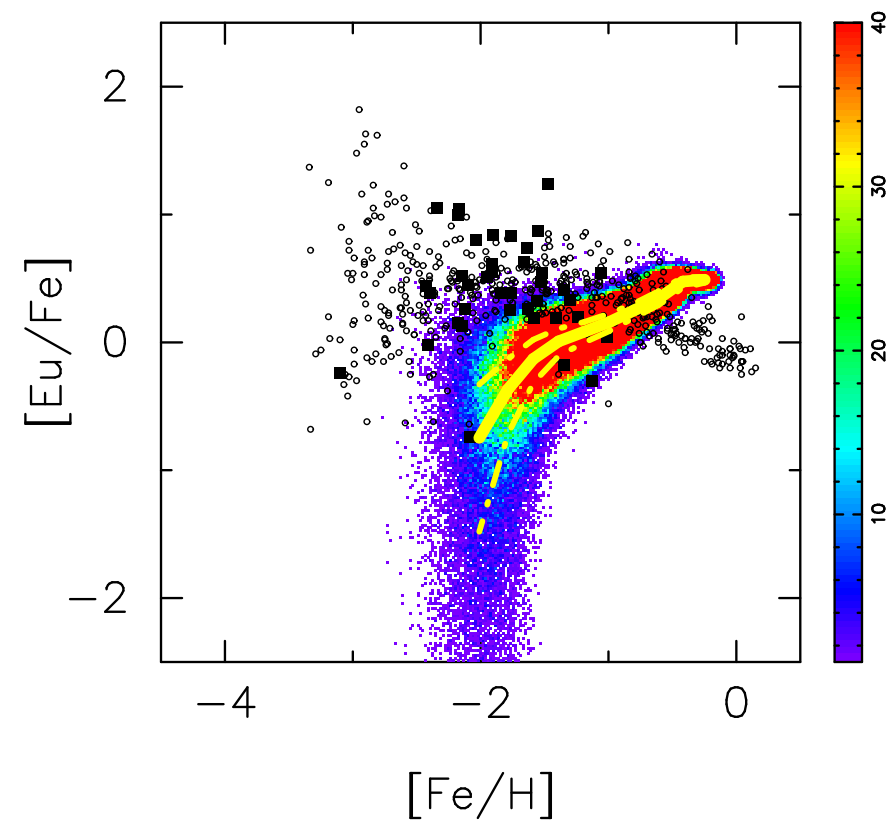

FIG. 10. - $[\mathrm{Eu} / \mathrm{Fe}]$ as a function of $[\mathrm{Fe} / \mathrm{H}]$ of model mExt $\left(M_{\text {tot }}\right.$ $=7 \times 10^{8} M_{\odot}, c_{\star}=0.033, n_{\mathrm{th}}=0.1 \mathrm{~cm}^{-3}, \epsilon_{\mathrm{SN}}=3 \times 10^{49} \mathrm{erg}$, and $t=100 \mathrm{Myr})$. Symbols are the same as Figure 5

\subsection{Merger time of neutron star mergers}

In this section, we discuss the effect of merger time of NSMs. Figure 11 shows resultant $[\mathrm{Eu} / \mathrm{Fe}]$ as a function of $[\mathrm{Fe} / \mathrm{H}]$ by NSMs with different merger time $\left(t_{\mathrm{NSM}}\right)$. 
Eu in Figure 11 (a) and (b) are produced by NSMs with $t_{\mathrm{NSM}}=10 \mathrm{Myr}(\mathrm{mt10})$ and $500 \mathrm{Myr}$ (mt500), respectively. Although mt10 has a slightly smaller fraction of stars in $-3<[\mathrm{Fe} / \mathrm{H}]<-2$ than model m000, the global relative abundance ratio is similar to $\mathrm{m} 000\left(t_{\mathrm{NSM}}=100\right.$ Myr). Contrary to the models m000 and mt10, the model with much longer merger time such as $500 \mathrm{Myr}$ in mt500 shows large scatters in $[\mathrm{Eu} / \mathrm{Fe}]$ at higher metallicity and cannot account for the observed scatters in $[\mathrm{Fe} / \mathrm{H}] \sim-3$.

Figure 12 shows $[\mathrm{Fe} / \mathrm{H}]$ as a function of the substantial galactic age, i.e., the elapsed time from the rise of the major star formation. As shown in Figure 3, we can regard that the major star formation arises from $600 \mathrm{Myr}$ from the beginning of the calculation. The average metallicity of stars is almost constant during the first $\sim 300 \mathrm{Myr}$. Due to low star formation efficiency of the galaxy, spatial distribution of metallicity is highly inhomogeneous in $\lesssim$ $300 \mathrm{Myr}$. In this epoch, since most of gas particles are enriched only by a single SN, metallicity of stars is mainly determined simply by the distance from each SN to the gas particles which formed the stars. Therefore, NSMs with $t_{\mathrm{NSM}} \sim 100 \mathrm{Myr}$ can account for the observation of EMP stars, as well as those with $t_{\mathrm{NSM}} \sim 10 \mathrm{Myr}$. In contrast, metallicity is well correlated with the galactic age after $\sim 300 \mathrm{Myr}$, irrespective of the distance from each $\mathrm{SN}$ to the gas particles. Because SN products have already been well mixed in a galaxy, the stellar metallicity is determined by the number of the SNe, which enriched the stellar ingredients. Therefore, if the merger time of NSMs is much longer than $\sim 300 \mathrm{Myr}$, it is too long to reproduce observations.

\subsection{The rate of neutron star mergers}

The yields of $r$-process elements in our models are related to the NSM rate as already mentioned in $₫ 2.2$, though the Galactic rate of NSMs is highly uncertain. The estimated Galactic NSM rate is $10^{-6}$ to $10^{-3} \mathrm{yr}^{-1}$ based on three observed binary pulsars Abadie et al. 2010a). Table5lists yields of models discussed here. Figure [13. shows predicted $[\mathrm{Eu} / \mathrm{Fe}]$ as a function of $[\mathrm{Fe} / \mathrm{H}]$ assuming different NSM rate. Figure 13 (a) and (b) represent models with the NSM fractions $f_{\mathrm{NSM}}=0.001$ $(\mathrm{mr} 0.001)$ and $f_{\mathrm{NSM}}=0.1$ (mr0.1), respectively. The corresponding NSM rate in a MW-like galaxy is $\sim 10^{-5} \mathrm{yr}^{-1}$ (mr0.001) and $\sim 10^{-3} \mathrm{yr}^{-1}(\mathrm{mr0.1})$. Model mr0.001 predicts larger scatter and a smaller number of stars at $[\mathrm{Fe} / \mathrm{H}]<-3$ than m000. Model mr0.001 has $[\mathrm{Eu} / \mathrm{Fe}]$ dispersion by more than 3 dex at $[\mathrm{Fe} / \mathrm{H}]=-2$. In addition, there remains $\sim 1$ dex dispersion even for stars with $[\mathrm{Fe} / \mathrm{H}]>-2$. In contrast, model mr0.1 predicts smaller scatter than m000, though it does not seem to be inconsistent with observations. Such tendencies are also seen in Argast et al. (2004), Komiva et al. (2014) and van de Voort et al. (2015).

Our fiducial model, m000, reproduces the observed $r$-process ratio as discussed in 4.2 The NSM rate of $\mathrm{m} 000$ for a MW-like galaxy is $\sim 10^{-4} \mathrm{yr}^{-1}$. The total mass of $r$-process elements produced by each NSM corresponds to $\sim 10^{-2} M_{\odot}$. The value is consistent with recent nucleosynthesis calculations: $10^{-3} M_{\odot}$ to $10^{-2} M_{\odot}$ (e.g., Goriely et al. 2011; Korobkin et al. 2012; Hotokezaka et al. 2013; Bauswein et al. 2013; Wanajo et al. 2014).

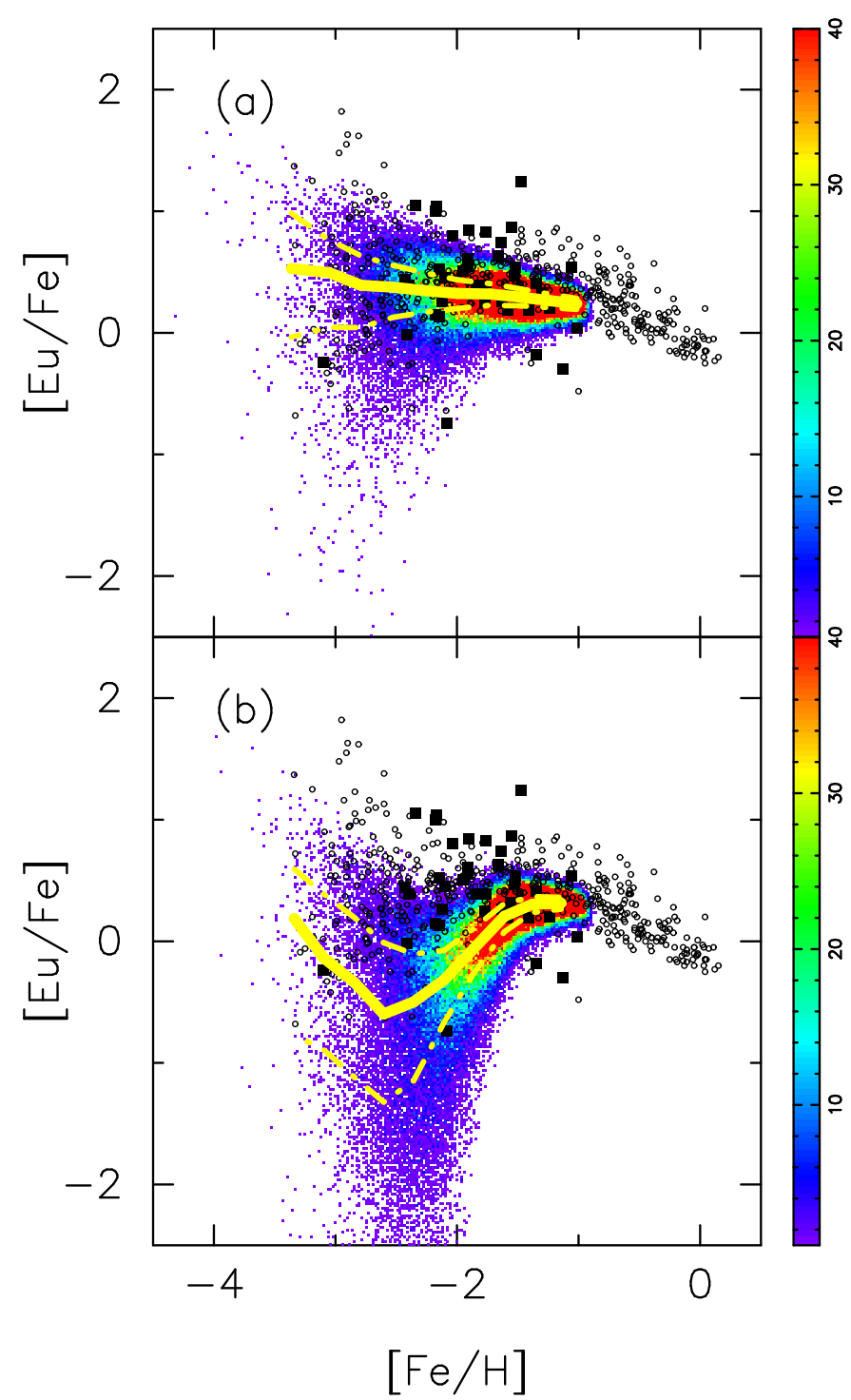

FIG. 11. - $[\mathrm{Eu} / \mathrm{Fe}]$ as a function of $[\mathrm{Fe} / \mathrm{H}]$ with different merger time of NSMs. (a): $\operatorname{mt10}\left(t_{\mathrm{NSM}}=10 \mathrm{Myr}\right)$. (b): $\operatorname{mt} 500\left(t_{\mathrm{NSM}}=\right.$ $500 \mathrm{Myr})$. Symbols are the same as Figure 5

Argast et al. (2004) construct an inhomogeneous chemical evolution model of the MW halo. Their model is difficult to reproduce $[\mathrm{Eu} / \mathrm{Fe}]$ by NSMs with the Galactic NSM rate of $2 \times 10^{-4} \mathrm{yr}^{-1}$ due to high star formation efficiency. $[\mathrm{Eu} / \mathrm{Fe}]$ produced in their model is similar to that of mExt (Figure 10).

From the discussion above, NSM rate of $\sim 10^{-4} \mathrm{yr}^{-1}$ in a MW size galaxy is preferred to reproduce the observed $[\mathrm{Eu} / \mathrm{Fe}]$. This rate is consistent with the estimated galactic NSM rate from the observed binary pulsars (Abadie et al. 2010a). Near future gravitational detectors, KAGRA, advanced LIGO, and advanced VIRGO Abadie et al. 2010b; Kuroda \& LCGT Collaboration 2010; Accadia et al 2011; LIGO Scientific Collaboration 2013) are expected to detect $10-100$ events per year of gravitational wave from NSMs.

\section{SUMMARY}




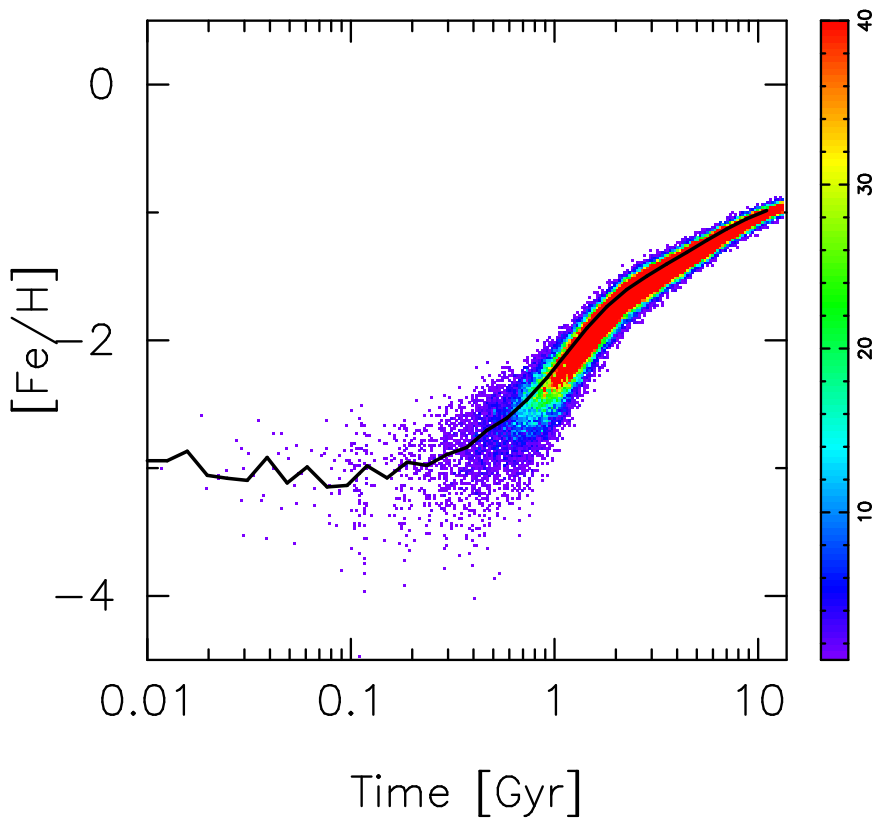

FIG. 12.- $[\mathrm{Fe} / \mathrm{H}]$ as a function of time of model m000. The horizontal axis is plotted from $600 \mathrm{Myr}$ from the start of the simulation. Black curve is the average of the metallicity in each age. Contour is the same as Figure 5

TABLE 5

LIST OF YIELDS.

\begin{tabular}{|c|c|c|}
\hline Model & $f_{\mathrm{NSM}}$ & $\begin{array}{c}M_{\mathrm{r}} \\
\left(M_{\odot}\right)\end{array}$ \\
\hline mr0.001 & 0.001 & $10^{-1}$ \\
\hline $\mathrm{m} 000$ & 0.01 & $10^{-2}$ \\
\hline mr0.1 & 0.1 & $10^{-3}$ \\
\hline \multicolumn{3}{|c|}{$\begin{array}{l}\text { NOTE. - The columns } \\
\text { correspond to the name of } \\
\text { models, fraction of NSMs } \\
\left(f_{\mathrm{NSM}}\right) \text {, and total yields } \\
\text { of } r \text {-process elements }\left(M_{\mathrm{r}}\right) \text {. } \\
\text { Fraction of NSMs is the } \\
\text { fraction of stars that cause } \\
\text { NSMs in the mass range } 8- \\
20 M_{\odot} \text {. }\end{array}$} \\
\hline
\end{tabular}

We have carried out numerical simulations of chemodynamical evolution of dSphs using $N$-body/SPH code, ASURA to investigate the enrichment history of $r$ process elements. This study suggests that NSMs with the merger time of $\sim 100 \mathrm{Myr}$ and the Galactic event rate of $\sim 10^{-4} \mathrm{yr}^{-1}$ can explain the dispersion of $[\mathrm{Eu} / \mathrm{Fe}]$ in reasonable agreement with observations in EMP stars. This study supports that NSMs are the major astrophysical site of the $r$-process. Our isolated dSph models reproduce basic properties of the observed LG dSphs such as radial profiles, time variations of the SFR as well as metallicity distribution. Here, we summarize the main results.

(1) The abundance ratio of $[\mathrm{Eu} / \mathrm{Fe}]$ produced in our models without metal mixing in star-forming regions has too large dispersion. This is because metals in a gas particle do not diffuse out to mix into the other particles throughout the evolution of galaxies.

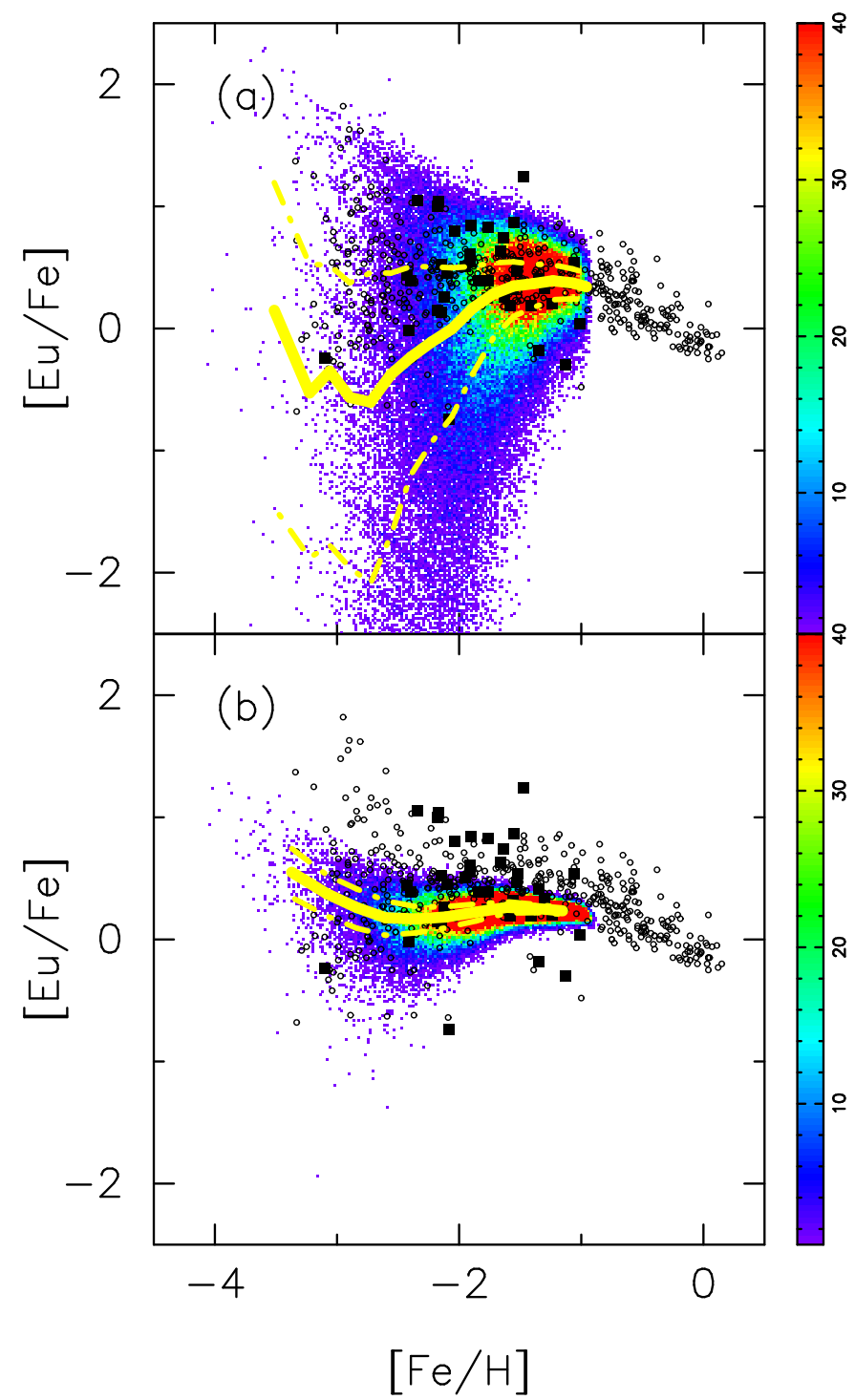

FIG. 13. - $[\mathrm{Eu} / \mathrm{Fe}]$ as a function of time with different rate of NSMs. (a): $\operatorname{mr} 0.001\left(f_{\mathrm{NSM}}=0.001\right) .(\mathrm{b}): \operatorname{mr} 0.1\left(f_{\mathrm{NSM}}=0.1\right)$. Symbols are the same as Figure 5

(2) Models with metal mixing in star-forming region reproduce the observed $[\mathrm{Eu} / \mathrm{Fe}]$ distribution and its scatter as a function of $[\mathrm{Fe} / \mathrm{H}]$. Our model shows good convergence of the resolution. We show that NSMs with $t_{\mathrm{NSM}}=100 \mathrm{Myr}$ is favorable for reproducing the observed $[\mathrm{Eu} / \mathrm{Fe}]$ as a function of metallicity. This result implies that the metal mixing process is critical to reproduce the $[\mathrm{Eu} / \mathrm{Fe}]$ distribution. In addition, this study suggests that the SFR of dSphs in the early epoch of their evolution $\sim 1 \mathrm{Gyr}$ is $\lesssim 10^{-3} M_{\odot} \mathrm{yr}^{-1}$.

(3) The NSMs with merger time of $\lesssim 300$ Myr is acceptable to account for the observed abundance of EMP stars. This is because metallicity is not correlated with time up to $\sim 300 \mathrm{Myr}$ from the start of the simulation due to low star formation efficiency of the model galaxy. 
(4) This study suggests that the Galactic NSM rate to account for the observed $r$-process abundance scatters is $\sim 10^{-4} \mathrm{yr}^{-1}$. The total mass of $r$ process elements ejected by one NSM is $\sim 10^{-2} M_{\odot}$, which is consistent with the value suggested by nucleosynthesis studies $\left(10^{-3}-10^{-2} M_{\odot}\right)$. Next generation gravitational detectors KAGRA, advanced LIGO and advanced VIRGO are expected to detect gravitational wave from NSMs and their event rate would be over 10 per year. Their detections will give us more reliable galactic NSM rate.

In this study, we have focused on the enrichment history of $r$-process elements in isolated dSphs with fixed mass. To fully understand the enrichment history of $r$ process elements in the LG galaxies, it is important to show how the mass and size of galaxies affect the enrichment of $r$-process elements.

Recent observations suggest that the low abundance of $r$-process elements $([\mathrm{Ba} / \mathrm{Fe} 3<-1)$ in stars with $[\mathrm{Fe} / \mathrm{H}]$ $<-3.5$. These stars provide clues to understand the astrophysical site(s) of $r$-process elements and the metal enrichment in the first galaxies. Some studies suggest that the $r$-process abundance of these stars can be explained by short merger time channel ( $\sim 1 \mathrm{Myr})$ of NSMs (Ishimaru et al. 2015) or accretion of materials from inter stellar medium to the Population III stars (Komiva et al. 2014). Since we have only focused on the star-to-star scatters of $[\mathrm{Eu} / \mathrm{Fe}]$ in stars with $[\mathrm{Fe} / \mathrm{H}] \sim-3$ assuming that NSMs are the major site of $r$-process in this paper, we have not discussed the origin of these stars. It is profitable to discuss the origin of these stars by the detailed simulation of galaxies.

To address all of these issues, it is required to understand how the MW halo formed. We need to clarify the relation between the building block galaxies of the MW and the present LG dSphs. It is now underway to extend our numerical simulations of chemodynamical evolution of dSphs to larger scale simulations of the MW in order to fully understand the enrichment of $r$-process elements in the MW and confirm the validity of the scenario of hierarchical structure formation.

We appreciate the referee for giving us profitable comments to improve our paper. We are grateful to Masaomi Tanaka, Shinya Wanajo, Grant J. Mathews, and Yuichiro Sekiguchi for fruitful discussion. We also appreciate for Takuma Suda for providing us the latest version of SAGA database including the dataset of the LG dSphs. This work is supported in part by MEXT SPIRE and JICFuS. YH is financially supported by Grant-in-Aid for JSPS Fellows (15J00548). The authors are financially supported by JSPS Grants-in-Aid for Scientific Research (YI: 26400232, TRS: 26707007, MSF: 26800108, TK: 26105517, 24340060, 15H03665). Numerical computations and analysis were in part carried out on Cray XC30 and computers at Center for Computational Astro-

$\S$ Barium (Ba) can also be regarded as the $r$-process element in $[\mathrm{Fe} / \mathrm{H}] \lesssim-3$ physics, National Astronomical Observatory of Japan.

\section{APPENDIX}

\section{A. PARAMETER DEPENDENCE}

\section{A.1. Radial profile}

We compare models with different threshold densities for star formation $\left(n_{\mathrm{th}}\right)$, dimensionless SFE parameters $\left(c_{\star}\right)$, and SN feedback energies $\left(\epsilon_{\mathrm{SN}}\right)$. Table A1 lists all models discussed here. Figure A1 shows the radial profiles of our models. Horizontal axis of Figure A1 is the distance from the galactic center. Figure A1 (a) shows the dark matter density profile. We find that all models have similar dark matter profiles. The dark matter profile is not affected by physical parameters such as threshold density for star formation, dimensionless SFE parameters, and SN feedback energy.

Gas density, stellar density, and stellar velocity dispersion profiles have variations among these models. The gas density of sn10 and se01 is lower than that of s000 (Figure A1 (b)). In these models, most of gas is consumed in the early phase of their evolution. The gas density of se01 is truncated at $0.3 \mathrm{kpc}$ while the gas profiles of the other models continue over $10 \mathrm{kpc}$. This is because the feedback energy in se01 is too weak to blow the gas away to the outer region of the galaxy.

Stellar density profiles for all models (Figure A1 (c)) are truncated within a few kpc, which is consistent with the observed truncation radius $(\sim 0.5-3 \mathrm{kpc})$ of dSphs in the LG (Irwin \& Hatzidimitriou 1995). In sn10, stars distribute to a larger radius than the other models. When a low value of $n_{\text {th }}$ is used, stars can form in the outer region of the galaxy. If the SN feedback is weak (model se01), stellar distribution concentrates on the central region of the galaxy.

Figure A1(d) shows the velocity dispersion profile. All models except for se01 are consistent with the observed radial velocity dispersion profiles inside $1 \mathrm{kpc}$ from the center in the LG dSphs (Walker et al. 2007, 2009). Model se01 has higher velocity dispersion at the center of our model galaxy due to the high central concentration of stars.

In contrast to $n_{\mathrm{th}}$ and $\epsilon_{\mathrm{SN}}$, the dimensionless $\mathrm{SFE}$ parameter $\left(c_{\star}\right)$ does not greatly affect the radial profiles. Figure A1 shows all profiles of s000 (red curve) and sc50 (blue curve) are similar although they have different value of the dimensionless SFE parameter. These features suggest that radial properties of galaxies are insensitive to the value of $c_{\star}$ when we adopt a reasonable value of $n_{\mathrm{th}}\left(=100 \mathrm{~cm}^{-3}\right)$ (Saitoh et al. 2008).

\section{A.2. Time variations of the star formation rate and metallicity distribution}

Figure $\mathrm{A} 2$ and $\mathrm{A} 3$ show SFR as a function of time and metallicity distribution, respectively. SFR as a function of time and metallicity distribution are characterized by the threshold density for star formation and SN feedback energy. Model sn10 has lower $n_{\text {th }}\left(=10 \mathrm{~cm}^{-3}\right)$ than that of s000 $\left(n_{\mathrm{th}}=100 \mathrm{~cm}^{-3}\right)$. The second peak of SFR of sn10 is earlier than s000. This reflects time required to reach $n_{\text {th }}$ is shorter than s000 because of using low $n_{\text {th }}$ value in sn10. In addition, the first peak of SFR of sn10 
TABLE A1

LIST OF MODELS.

\begin{tabular}{lrll}
\hline \hline Model & $\begin{array}{c}n_{\text {th }} \\
\mathrm{cm}^{-3}\end{array}$ & \multicolumn{1}{c}{$c_{\star}$} & $\begin{array}{l}\epsilon_{\mathrm{SN}} \\
10^{51} \mathrm{erg}\end{array}$ \\
\hline s000 & 100 & 0.033 & 1 \\
sn10 & 10 & 0.033 & 1 \\
sc50 & 100 & 0.5 & 1 \\
se01 & 100 & 0.033 & 0.1 \\
\hline
\end{tabular}

Note. - Parameters adopted in our models: (1) Model: Name of our models. (2) $c_{\star}$ : Dimensionless star formation efficiency parameter. (3) $n_{\text {th }}$ :

Threshold density for star formation.

(4) $\epsilon_{\mathrm{SN}}: \mathrm{SN}$ feedback energy.
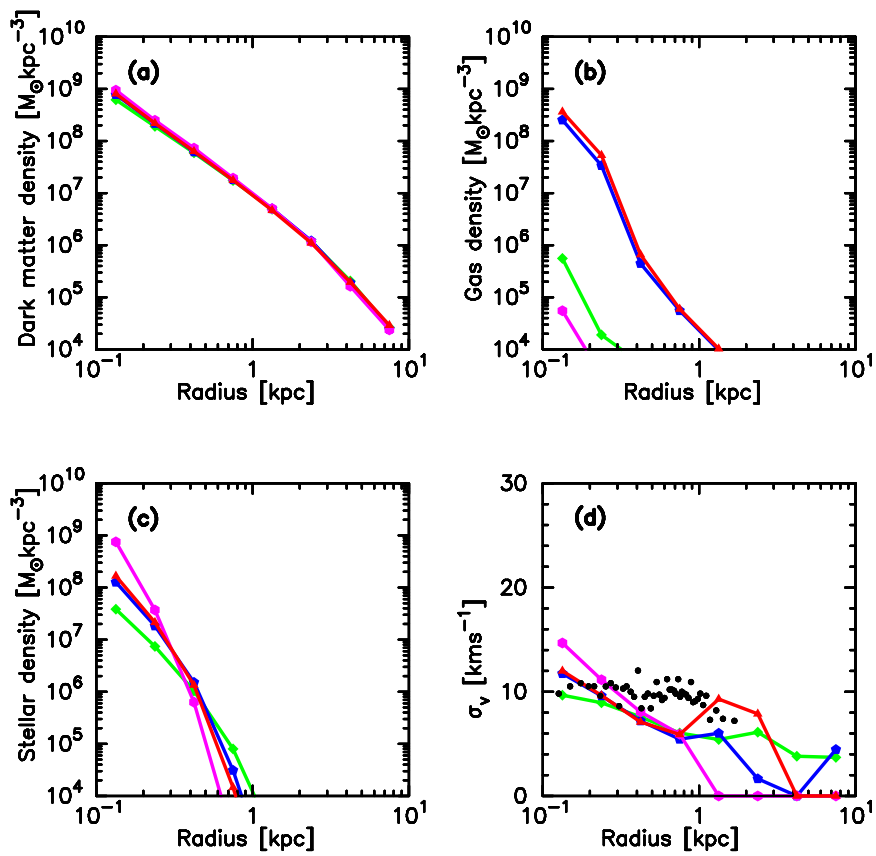

Fig. A1. - Radial profiles of models of different parameters at $t=5.0$ Gyr. Red triangles, green diamonds, blue pentagons, and magenta hexagons represent model s000, sn10 $\left(n_{\mathrm{th}}=10 \mathrm{~cm}^{-3}\right)$, c050s $\left(c_{\star}=0.5\right)$, and se01 $\left(\epsilon_{\mathrm{SN}}=10^{50} \mathrm{erg}\right)$, respectively. (a): Radial dark matter density profile. (b): Radial gas density profile. (c): Radial stellar density profile. (d): Radial velocity dispersion profile.

is higher than s000. Gas is consumed by star formation and removed by outflow at $<0.1$ Gyr in sn10. Its SFR is therefore $\sim 2$ dex lower than s000. The early bursty star formation of sn10 produces more metal-poor stars than s000 (see green dashed and red curves in Figure A3). Higher SFR of sn10 0.1 Gyr produces more CCSNe in this phase. CCSNe produce outflow. Model sn10 thus loses larger amount of gas around $\sim 0.1$ Gyr. Chemical evolution of sn10, therefore, quenches at $>0.1$ Gyr and produces only few metal-rich stars.

SN feedback energy also significantly affects the time variation of SFR and metallicity distribution. The SN feedback energy of se01 $\left(\epsilon_{\mathrm{SN}}=10^{50} \mathrm{erg}\right)$ is ten times smaller than that of $\mathrm{s} 000\left(\epsilon_{\mathrm{SN}}=10^{51} \mathrm{erg}\right)$. The peak of the SFR of se01 is over 1 dex higher than that of s000. SN feedback energy gives thermal energy to gas particles. It prevents collapse of gas particles. As a result, star formation is suppressed due to SN feedback energy.
SN feedback energy of se01 is too weak to suppress star formation. The peak of SFR of se01 is thus $\sim 1$ dex higher than s000. Gas is consumed for star formation due to high SFR around 2 Gyr of se01, and the SFR at $>4 \mathrm{Gyr}$ is eventually suppressed. Due to the low SN feedback energy in model se01, the peak of metallicity distribution of se01 is $\sim 0.5$ dex higher metallicity than s000.

On the other hand, the value of the dimensionless SFE parameter does not significantly affect the results. Models s000 and sc50 have different value of $c_{\star}=0.033$ and 0.5 , respectively. The time variation of the SFR and metallicity distribution of sc50 is however similar to that of s000. This result suggests that the time variation of the SFR and metallicity distribution are insensitive to the value of $c_{\star}$ if we use a reasonable value of $n_{\mathrm{th}}(=100$ $\mathrm{cm}^{-3}$ ). Slightly lower metallicity of sc50 than s000 is due to slightly lower SFR of sc50 than s000. This result suggests that the value of $c_{\star}$ does not affect the metallicity distribution as well as radial profiles and the SFR. When we adopt $n_{\mathrm{th}}=100 \mathrm{~cm}^{-3}$, it takes much longer local dynamical time to flow from the reservoir $\left(n_{\mathrm{H}} \sim 1 \mathrm{~cm}^{-3}\right)$ to the star-forming regions $\left(n_{\mathrm{H}} \gtrsim 100 \mathrm{~cm}^{-3}\right)$. This timescale does not depend on $c_{\star}$ (Saitoh et al. 2008). Our results are, thus, independent of $c_{\star}$.

These results suggest that the time variation of the SFR and metallicity distribution is significantly affected by the threshold density for star formation and SN feedback energy. Low $n_{\text {th }}$ model (sn10) produces too many EMP stars. In contrast, low $\epsilon_{\mathrm{SN}}$ model (se01) has too many metal-rich stars. These differences in metallicity distribution are due to the difference of the time variation of the SFR among models. On the other hand, model s000 reproduces observation of metallicity distribution as well as dynamical properties. Parameters of s000 are taken from the observed values. Threshold density of $\mathrm{s} 000\left(n_{\mathrm{th}}=100 \mathrm{~cm}^{-3}\right)$ is taken from mean density of GMCs. SN feedback energy of $\mathrm{s} 000\left(\epsilon_{\mathrm{SN}}=10^{51} \mathrm{erg}\right)$ is taken from the canonical explosion energy of CCSNe (e.g., Nomoto et al. 2006). We thus treat s000 as a model that has fiducial parameter sets. 


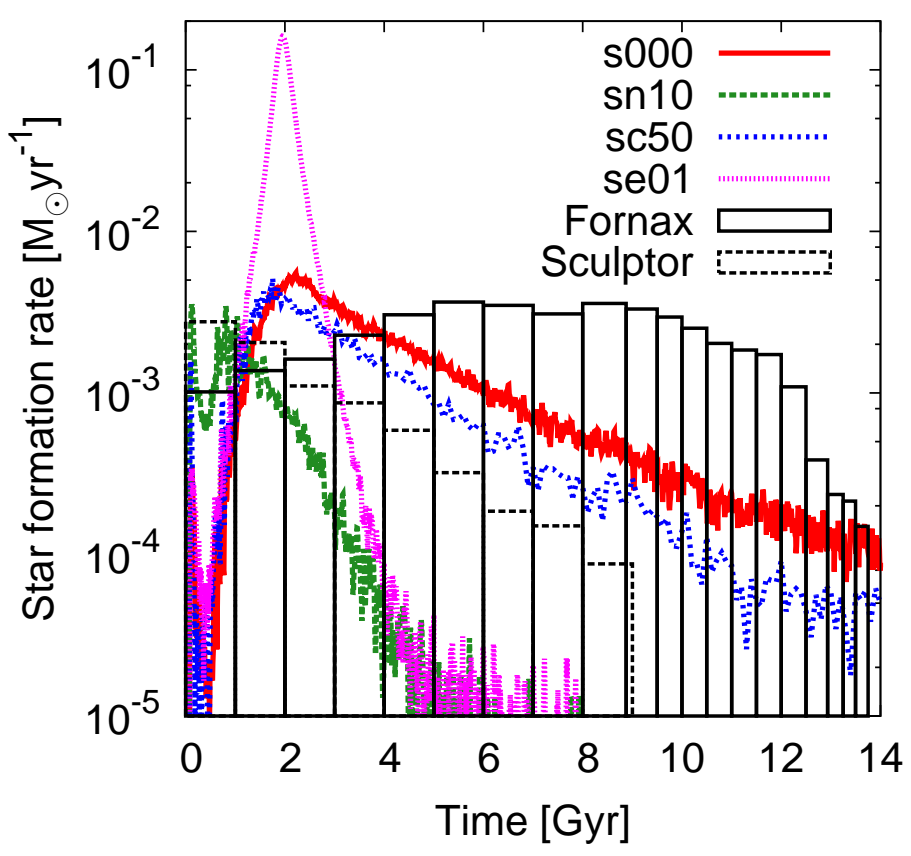

FIG. A2.- The SFR as a function of time for our models. Red curve, green dashed curve, blue short-dashed curve, and magenta dotted curve represent s000, $\operatorname{sn} 10\left(n_{\mathrm{th}}=10 \mathrm{~cm}^{-3}\right), \mathrm{sc} 50\left(c_{\star}=0.5\right)$, and se01 $\left(\epsilon_{\mathrm{SN}}=10^{50} \mathrm{erg}\right)$, respectively. The black histogram and black-dotted histogram represent the observed SFR of the Fornax dSph (de Boer et al. 2012b) and the Sculptor dSph (de Boer et al. 2012a), respectively.

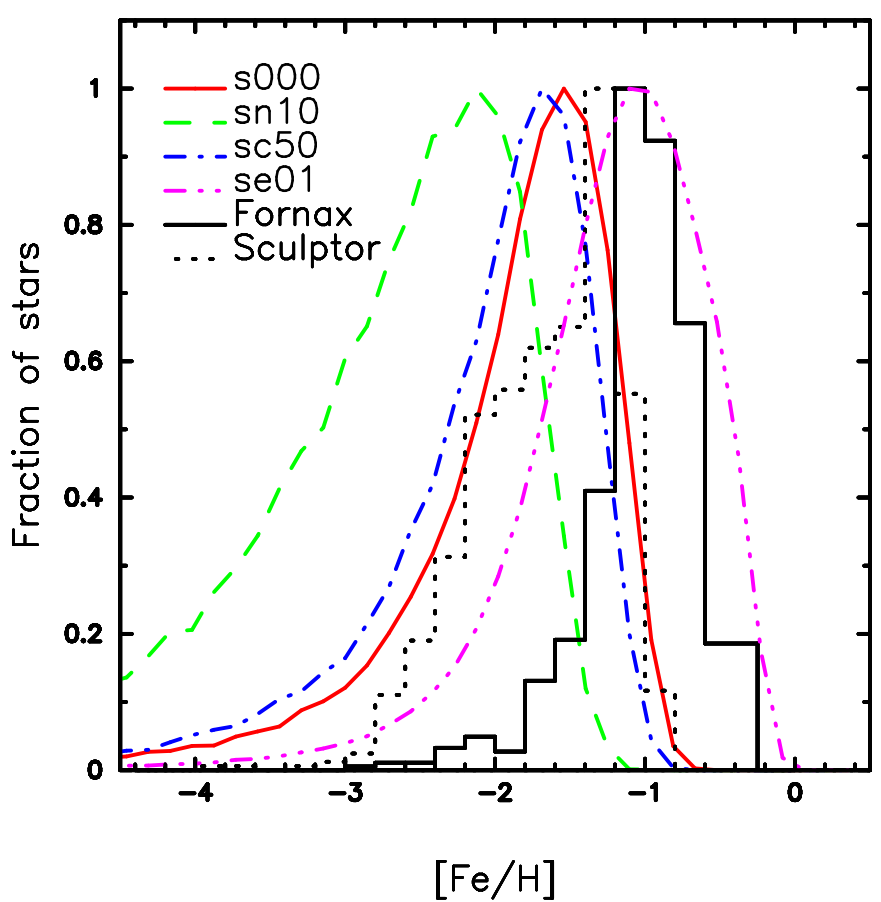

FIG. A3.- Metallicity distribution of our models. The black histogram and black-dotted histogram are observed metallicity distribution of the Fornax dSph (Kirby et al. |2010) and the Sculptor dSph (Kirby et al. 2009, 2010; Kirby \& Cohen 2012), respectively. Red curve, green dashed curve, blue dash-dotted curve, and magenta dash-dot-dotted curve represent $\mathrm{s} 000, \operatorname{sn} 10\left(n_{\mathrm{th}}=10 \mathrm{~cm}^{-3}\right)$, $\operatorname{sc} 50\left(c_{\star}=0.5\right)$, and $\operatorname{se} 01\left(\epsilon_{\mathrm{SN}}=10^{50} \mathrm{erg}\right)$, respectively.

\section{REFERENCES}

Abadie, J., Abbott, B. P., Abbott, R. et al. 2010a, CQGra, 27, 173001

Abadie, J., Abbott, B. P., Abbott, R., et al. 2010b, NIMPA, 624, 223

Accadia, T., Acernese, F., Antonucci, F., et al. 2011, CQGra, 28, 114002

Aoki, W., Suda, T., Boyd, R. N., Kajino, T., \& Famiano, M. A. 2013, ApJ, 766, L13

Argast, D., Samland, M., Gerhard, O. E., \& Thielemann, F.-K. 2000, A\&A, 356, 873

Argast, D., Samland, M., Thielemann, F.-K., \& Qian, Y.-Z. 2004, A\&A, 416, 997

Barnes, J. \& Hut, P. 1986, Nature, 324, 446

Bauswein, A., Goriely, S., \& Janka, H.-T. 2013, ApJ, 773, 78

Berger, E., Fong, W., \& Chornock, R. 2013, ApJ, 774, L23

Binney, J., \& Tremaine, S. 2008, Galactic dynamics (2nd ed., Princeton, NJ: Princeton University Press)

Bubar, E. J., \& King, J. R., 2010, AJ, 140, 293

Burris, D. L., Pilachowski, C. A., Armandroff, T. E., et al. 2000, ApJ, 544, 302

Cescutti, G., Romano, D., Matteucci, F., Chiappini, C., \&

Hirschi, R. 2015, A\&A, 577, A139

Cioffi, D. F., McKee, C. F., \& Bertschinger, E. 1988, ApJ, 334, 252

de Boer, T. J. L., Tolstoy, E., Hill, V., et al. 2012a, A\&A, 539, A103

de Boer, T. J. L., Tolstoy, E., Hill, V., et al. 2012b, A\&A, 544, A73

De Silva, G. M., Freeman, K. C., Asplund, M., et al. 2007a, AJ, 133,1161

De Silva, G. M., Freeman, K. C., Bland-Hawthorn, J., Asplund, M., \& Bessell, M. S. 2007b, AJ, 133, 694

De Silva, G. M., Freeman, K. C., Bland-Hawthorn, J., et al. 2011 MNRAS, 415, 563

De Silva, G. M., D’Orazi, V., Melo, C., et al. 2013, MNRAS, 431, 1005

Dominik, M., Belczynski, K., Fryer, C., et al. 2012, ApJ, 759, 52
Eichler, D., Livio, M., Piran, T., \& Schramm, D. N. 1989, Nature, 340,126

Feng, Y. \& Krumholz, M. R. 2014, Nature, 513, 523

Ferland, G. J., Korista, K. T., Verner, D. A., et al. 1998, PASP, 110,761

Ferland, G. J., Porter, R. L., van Hoof, P. A. M, et al. 2013,

RMxAA, 49, 137

Fioc, M., \& Rocca-Volmerange, B. 1997, A\&A, 326, 950

François, P., Depagne, E., Hill, V. et al. 2003, A\&A, 403, 1105

Francois, P., Depagne, E., Hill, V. et al. 2007, A\&A, 476, 935

Frebel, A., Kirby, E. N., \& Simon, J. D. 2010a, Nature, 464, 72

Frebel, A., Simon, J. D., Geha, M., \& Willman, B. 2010b, ApJ, 708,560

Frebel, A., \& Norris, J. E. 2015, ARA\&A, 53, 631

Freiburghaus, C., Rosswog, S., \& Thielemann, F.-K. 1999, ApJ, 525, L121

Fulbright, J. P. 2000, AJ, 120, 1841

Gingold, R. A., \& Monaghan, J. J. 1977, MNRAS, 181, 375

Goriely, S., Bauswein, A., \& Janka, H.-T. 2011, ApJ, 738, L32

Goswami, A. \& Prantzos, N. 2000, A\&A, 359, 191

Harris, W. E., \& Pudritz, R. E. 1994, ApJ, 429, 177

Heyer, M., Krawczyk, C., Duval, J., \& Jackson, J. M. 2009, ApJ, 699,1092

Hernquist, L. 1993 ApJS, 86, 389

Honda, S., Aoki, W., Kajino, T., et al. 2004, ApJ, 607, 474

Horowitz, C. J., Shen, G., O'Connor, E., \& Ott, C. D.' 2012, Phys. Rev. C, 86, 065806

Hotokezaka, K., Kiuchi, K., Kyutoku, K., et al. 2013, Phys. Rev. D, 87, 024001

Hopkins, P. F., Quataert, E., \& Murray, N. 2011, MNRAS, 417, 950

Ishigaki, M. N., Aoki, W., \& Chiba, M. 2013, ApJ, 771, 67

Ishimaru, Y., \& Wanajo, S. 1999, ApJ, 511, L33

Ishimaru, Y., Wanajo, S., Aoki, W., \& Ryan, S. G. 2004, ApJ, 600, L47

Ishimaru, Y., Wanajo, S., \& Prantzos, N. 2015, ApJ, 804, L35

Irwin, M., \& Hatzidimitriou, D. 1995, MNRAS, 277, 1354

Johnson, J. A. 2002, ApJS, 139, 219 
Katz, N. 1992, ApJ, 391, 502

Katz, N., Weinberg, D. H., \& Hernquist, L. 1996, ApJS, 105, 19

Kauffmann, G., White, S. D. M., \& Guiderdoni, B. 1993, MNRAS, 264, 201

Kawata, D. 2001, ApJ, 558, 598

Kinugawa, T., Inayoshi, K., Hotokezaka, K., Nakauchi, D., \& Nakamura, T., 2014, MNRAS, 442, 2963

Kirby, E. N., \& Cohen, J. G. 2012, AJ, 144, 168

Kirby, E. N., Cohen, J. G., Guhathakurta, P., et al. 2013, ApJ, 779,102

Kirby, E. N., Guhathakurta, P., Bolte, M., Sneden, C., \& Geha M. C. 2009, ApJ, 705, 328

Kirby, E. N., Guhathakurta, P., Simon, J. D., et al. 2010, ApJS, 191, 352

Kobayashi, C., \& Nakasato, N. 2011, ApJ, 729, 16

Komiya, Y., Yamada, S., Suda, T., \& Fujimoto, M. Y. 2014, ApJ 783,132

Korobkin, O., Rosswog, S., Arcones, A., \& Winteler, C. 2012, MNRAS, 426, 1940

Krumholz, M. R., \& Tan, J. C. 2007, ApJ, 654, 304

Kuroda, K., \& LCGT Collaboration 2010, CQGra, 27, 084004

Lada, C. J., \& Lada, E. A. 2003, ARA\&A, 41, 57

Larson, R. B. 1981, MNRAS, 194, 809

Lattimer, J. M., \& Schramm, D. N. 1974, ApJ, 192, L145

Lattimer, J. M. \& Schramm, D. N. 1976, ApJ, 210, 549

Lattimer, J. M., Mackie, F., Ravenhall, D. G., \& Schramm, D. N. 1977, ApJ, 213, 225

Lemasle, B., Hill, V., Tolstoy, E., et al. 2012, A\&A, 538, A100

Letarte, B., Hill, V., Tolstoy, E., et al. 2010, A\&A, 523, A17

LIGO Scientific Collaboration 2013, arXiv: 1304.0670

Liszt, H. S., Delin, X., \& Burton, W. B. 1981, ApJ, 249, 532

Lorimer, D. R. 2008, LRR, 11, 8

Lucy, L. B. 1977, AJ, 82, 1013

Makino, J. 1991, PASJ, 43, 621

Maoz, D., Mannucci, F. 2012, PASA, 29, 447

Mennekens, N., \& Venbeveren, D. 2014, A\&A, 564, A134

Meyer, B. S. 1989, ApJ, 343, 254

Mayer, L., Mastropietro, C., Wadsley, J., Stadel, J., \& Moore, B. 2006, MNRAS, 369, 1021

Martínez-Pinedo, G., Fischer, T., Lohs, A., \& Huther, L. 2012, Phys. Rev. Lett., 109, 251104

Mathews, G. J., \& Cowan, J. J. 1990, Nature, 345, 491

Mathews, G. J., Bazan, G., \& Cowan, J. J. 1992, ApJ, 391, 719

Matteucci, F., Romano, D., Arcones, A., Korobkin, O., \& Rosswog, S. 2014, MNRAS, 438, 2177

McConnachie, A. W. 2012, A.J, 144, 4

McWilliam, A., Preston, G. W., Sneden, C., \& Leonard, S. 1995, ApJ, 109, 2757

McWilliam, A. 1998, AJ, 115, 1640

Meyer, B. S., Mathews, G. J., Howard, W. M., Woosley, S. E., \& Hoffman, R. D. 1992, ApJ, 399, 656

Monaghan, J. J. 1992, ARA\&A, 30, 543

Monaghan, J. J., \& Lattanzio, J. C. 1985, A\&A, 149, 135

Moore, B., Ghigna, S., Governato, F., et al. 1999, ApJ, 524, L19

Navarro, J. F., \& White, S. D. M. 1993, MNRAS, 265, 271

Nichols, M., Revaz, Y., \& Jablonka, P. 2014, A\&A, 564, A112

Nishimura, N., Takiwaki, T., \& Thielemann, F.-K. 2015, ApJ, 810,109

Nomoto, K., Tominaga, N., Umeda, H., Kobayashi, C., \& Maeda, K. 2006, Nucl. Phys. A, 777, 424

Norris, J. E., Ryan, S. G., \& Beers, T. C. 2001, ApJ, 561, 1034

Okamoto, T., Eke, V. R., Frenk, C. S., \& Jenkins, A. 2005 MNRAS, 363, 1299

Okamoto, T., Jenkins, A., Eke, V. R., Quilis, V., \& Frenk, C. S. 2003, MNRAS, 345, 429

Okamoto, T., Nemmen, R. S., \& Bower, R. G. 2008, MNRAS, 385,161

Pancino, E., Carrera, R., Rossetti, E. \& Gallart, C. 2010, A\&A, 511, A56

Planck Collaboration XVI 2014, A\&A, 571, A16

Portegies Zwart, S. F., \& Yungelson, L., R., A\&A, 332, 173

Prantzos, N. 2008, A\&A, 489, 525

Qian, Y.-Z. 2000, ApJ, 534, L67

Qian, Y.-Z., \& Woosley, S. E. 1996, ApJ, 471, 331

Raiteri, C. M., Villata, M., Gallino, R., Busso, M., \& Cravanzola, A. 1999, ApJ, 518, L91

Reddy, A. B. S., Giridhar, S. \& Lambert, D. L. 2012, MNRAS, 419,1350
Reddy, A. B. S., Giridhar, S. \& Lambert, D. L. 2013, MNRAS, 431, 3338

Reddy, S., Prakash, M., \& Lattimer, J. M. 1998, Phys. Rev. D, 58, 013009

Revaz, Y., \& Jablonka, P. 2012, A\&A, 538, A82

Revaz, Y., Jablonka, P., Sawala, T., et al. 2009, A\&A, 501, 189

Roberts, L. F. 2012, ApJ, 755, 126

Roberts, L. F., Reddy, S., \& Shen, G. 2012, Phys. Rev. C, 86, 065803

Rosswog, S., Korobkin, O., Arcones, A., Thielemann, F.-K., \& Piran, T. 2014, MNRAS, 439, 744

Ryan, S. G., Norris, J. E., \& Beers, T. C. 1996, ApJ, 471, 254

Saitoh, T. R., Daisaka, H., Kokubo, E., et al. 2008, PASJ, 60, 667

Saitoh, T. R., Daisaka, H., Kokubo, E., et al. 2009, PASJ, 61, 481

Saitoh, T. R., \& Makino, J. 2009, ApJ, 697, L99

Saitoh, T. R., \& Makino, J. 2010, PASJ, 62, 301

Salpeter, E. E. 1955, ApJ, 121, 161

Sanders, D. B., Scoville, N. Z., \& Solomon, P. M. 1985, ApJ, 289, 373

Shen, S., Cooke, R., Ramirez-Ruiz, E., Madau, P., Mayer, L., \& Guedes, J. 2015, ApJ, 807, 115

Shen, S., Wadsley, J., \& Stinson, G. 2010, MNRAS, 407, 1581

Shetrone, M. D. 1996, A.J, 112, 1517

Sneden C., Cowan, J. J., \& Gallino, R. 2008, ARA\&A, 46, 241

Solomon, P. M., Rivolo, A. R., Barrett, J., \& Yahil, A. 1987, ApJ, 319,730

Steinmetz, M., \& Müller, E., 1994, A\&A, 281, L97

Stinson, G., Seth, A., Katz, N.,Wadsley, J., Governato, F., \& Quinn, T. 2006, MNRAS, 373, 1074

Suda, T., Katsuta, Y., Yamada, S., et al. 2008, PASJ, 60, 1159

Suda, T., Yamada, S., Katsuta, Y., et al. 2011, MNRAS, 412, 843

Suda, T., Hidaka, J., Ishigaki, M., et al. 2014,

Mem. Soc. Astron. Italiana, 85, 600

Symbalisty, E., \& Schramm, D. N. 1982, ApL, 22, 143

Tanaka, M., \& Hotokezaka, K. 2013, ApJ, 775, 113

Tanikawa, A., Yoshikawa, K., Okamoto, T., \& Nitadori, K. 2012 New A, 17, 82

Tanvir, N. R., Levan, A. J., Fruchter, A. S., et al. 2013, Nature, 500,547

Thornton, K., Gaudlitz, M., Janka, H. -Th., \& Steinmetz, M. 1998, ApJ, 500, 95

Ting, Y. -S., De Silva, G. M., Freeman, K. C., \& Parker, S. J. 2012, MNRAS, 427, 882

Tolstoy, E., Hill, V., \& Tosi, M. 2009, ARA\&A, 47, 371

Tsujimoto, T., \& Shigeyama, T. 2014, A\&A, 565, L5

Tsujimoto, T., Shigeyama, T., \& Yoshii, Y. 2000, ApJ, 531, L33

Travaglio, C., Galli, D., Gallino, R., Busso, M., Ferrini, F., Straniero, O. 1999, ApJ, 521, 691

Travaglio, C., Galli, D., \& Burkert, A. 2001, ApJ, 547, 217

van de Voort, F., Quataert, E., Hopkins, P. F., Kereš, D., \& Faucher-Giguère, C.-A. 2015, MNRAS, 447, 140

Walker, M. G., Mateo, M., Olszewski, E. W., et al. 2007, ApJ, 667, L53

Walker, M. G., Mateo, M., Olszewski, E. W., et al. 2009, ApJ, 704,1274

Wanajo, S. 2013, ApJ, 770, L22

Wanajo, S., Janka, H-T., \& Müller, B. 2011, ApJ, 726, L15

Wanajo, S., Kajino, T., Mathews, G. J., \& Otsuki, K. 2001, ApJ, 554,578

Wanajo, S., Sekiguchi, Y., Nishimura, N., et al. 2014, ApJ, 789, L39

Wehmeyer, B., Pignatari, M., \& Thielemann, F.-K., 2015 MNRAS, 452, 1970

Westin, J., Sneden, C., Gustafsson, B., \& Cowan, J. J. 2000, ApJ, 530,783

Wiersma, R. P. C., Schaye, J., Theuns, T., Vecchia, C. D., \& Tornatore, L. 2009, MNRAS, 399, 574

Winteler, C., Käppeli, R., Perego, A. et al. 2012, ApJ, 750, L22

Woolf, V. M., Tomkin, J., \& Lambert, D. L. 1995, ApJ, 453, 660

Woosley, S. E., Wilson, J. R., Mathews, G. J., Hoffman, R. D., \&

Meyer, B. S. 1994, ApJ, 433, 229

Yamada, S., Suda, T., Komiya, Y., Aoki, W., \& Fujimoto, M. Y., 2013, MNRAS, 436, 1362

Zuckerman, B., \& Evans, N. J., II. 1974, ApJ, 192, L149 